\title{
Conosiligins A-D, Ring-Rearranged Tremulane Sesquiterpenoids from Conocybe siliginea
}

Juan He,$^{\dagger}$ Chao-Jun Pu, ${ }^{\dagger}$ Meng Wang, ${ }^{\dagger}$ Zheng-Hui Li, ${ }^{\dagger}$ Tao Feng, ${ }^{* \dagger}$ Da-Ke Zhao, ${ }^{* \dagger}$ and Ji-Kai Liu, ${ }^{* \dagger}$

†School of Pharmaceutical Sciences, South-Central University for Nationalities, Wuhan 430074, People’s Republic of China

*Biocontrol Engineering Research Center of Plant Disease and Pest; Biocontrol Engineering Research Center of Crop Disease and Pest; School of Ecology and Environmental Science, Yunnan University, Kunming 650504, People's Republic of China

E-mail:tfeng@mail.scuec.edu.cn,liujikai@mail.scuec.edu.cn,zhaodk2012@ynu.edu.cn

\section{List of Supporting Information}

Section S1. ECD calculations for 1-4.

S1.1. Computational details for compound 1 (ECD)

S1.2. Computational details for compound 2 (ECD)

S1.3. Computational details for compound 3 (ECD)

S1.4. Computational details for compound 4 (ECD)

Section S2. Immunosuppressive Tests of 1-4

Section S3. NMR spectra for 1-4.

S3.1. NMR spectra (methanol-d $d_{4}$ ) and HRESIMS for conosiligin A (1)

S3.2. NMR spectra $\left(\mathrm{CDCl}_{3}\right)$ and HRESIMS for conosiligin B (2)

S3.3. NMR spectra (methanol-d $d_{4}$ ) and HRESIMS for conosiligin C (3)

S3.4. NMR spectra (methanol-d $d_{4}$ ) and HRESIMS for conosiligin D (4) 
Section S1. ECD calculations for 1-4.

\section{S1.1. Computational details for compound 1 (ECD)}

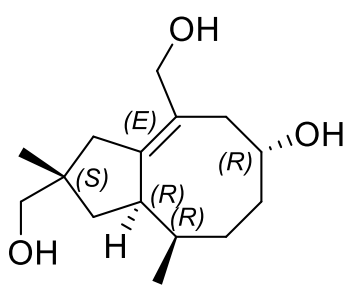

$1 \mathrm{a}$

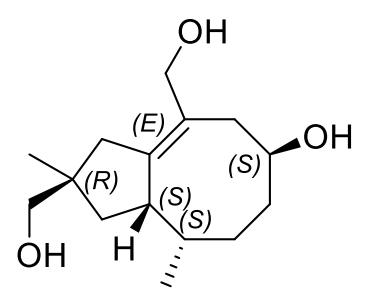

$1 \mathrm{~b}$

Conformation search based on molecular mechanics with MMFF94S force fields were performed for $\mathbf{1 a}$ and $\mathbf{1 b}$ gave 18 and 25 stable conformers, respectively. All these conformers were further optimized by the density functional theory method at the B3LYP/6-31G(d) level by Gaussian 16 program package. The ECD were calculated using density functional theory (TDDFT) at B3LYP/6-31+G(d) level in methanol with IEFPCM model. The calculated ECD curves were all generated using SpecDis 1.71 with $\sigma=0.22 \mathrm{eV}$, and UV shift $-19 \mathrm{~nm}$, respectively.

Figure S1. Comparison of the calculated ECD spectra for $\mathbf{1 a}$ and $\mathbf{1 b}$ with the experimental spectrum of $\mathbf{1}$ in methanol with PCM model.

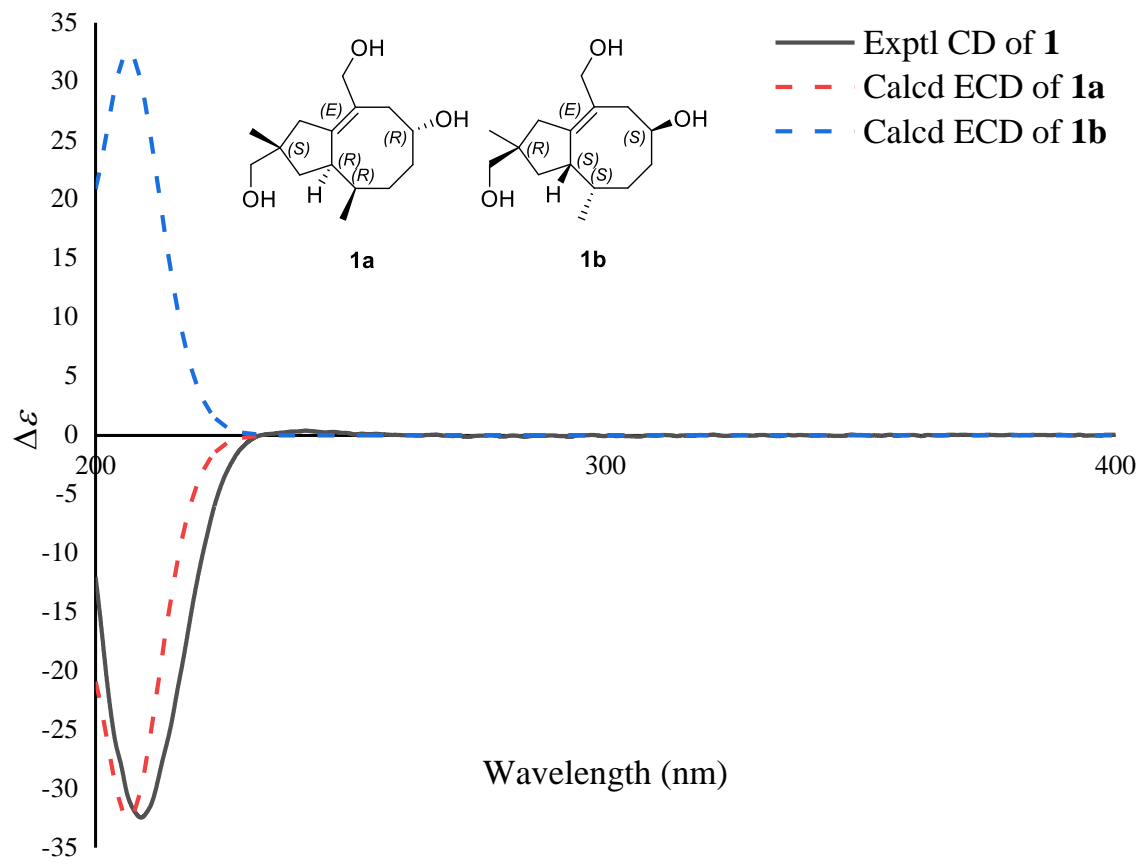


Table S1. Energy analysis for conformers of 1aA-1aR at B3LYP/6-31G(d) level in the gas phase

\begin{tabular}{|c|c|c|c|c|c|c|c|}
\hline Species & $E^{\prime}=E+Z P E$ & E & $H$ & G & $\Delta G$ & $\Delta E(\mathrm{kcal} / \mathrm{mol})$ & $P E \%$ \\
\hline $\mathbf{1 a A}$ & -812.491592 & -812.473108 & -812.472164 & -812.535768 & 0.000075 & 0.047063 & $17.09 \%$ \\
\hline $1 \mathbf{a B}$ & -812.491691 & -812.473225 & -812.472281 & -812.535843 & 0 & 0 & $18.50 \%$ \\
\hline $1 \mathrm{aC}$ & -812.489315 & -812.470225 & -812.469281 & -812.534989 & 0.000854 & 0.535893 & $7.48 \%$ \\
\hline 1aD & -812.488971 & -812.469799 & -812.468855 & -812.534901 & 0.000942 & 0.591114 & $6.82 \%$ \\
\hline $\mathbf{1 a E}$ & -812.487717 & -812.468436 & -812.467492 & -812.534016 & 0.001827 & 1.146460 & $2.67 \%$ \\
\hline $1 \mathrm{aF}$ & -812.489202 & -812.469961 & -812.469017 & -812.535235 & 0.000608 & 0.381526 & $9.71 \%$ \\
\hline $1 \mathrm{aG}$ & -812.487352 & -812.468021 & -812.467076 & -812.533579 & 0.002264 & 1.420682 & $1.68 \%$ \\
\hline 1aH & -812.483079 & -812.463640 & -812.462696 & -812.530285 & 0.005558 & 3.487698 & $0.05 \%$ \\
\hline 1aI & -812.483350 & -812.463937 & -812.462993 & -812.530497 & 0.005346 & 3.354666 & $0.06 \%$ \\
\hline 1aJ & -812.483986 & -812.464611 & -812.463667 & -812.531082 & 0.004761 & 2.987573 & $0.12 \%$ \\
\hline $1 \mathrm{aK}$ & -812.484128 & -812.464778 & -812.463834 & -812.531305 & 0.004538 & 2.847638 & $0.15 \%$ \\
\hline 1aL & -812.484210 & -812.464860 & -812.463916 & -812.531185 & 0.004658 & 2.922939 & $0.13 \%$ \\
\hline $1 \mathrm{aM}$ & -812.490636 & -812.472035 & -812.471091 & -812.535235 & 0.000608 & 0.381526 & $9.71 \%$ \\
\hline $1 \mathrm{aN}$ & -812.488727 & -812.469503 & -812.468559 & -812.534838 & 0.001005 & 0.630647 & $6.38 \%$ \\
\hline $1 \mathrm{aO}$ & -812.489551 & -812.470452 & -812.469508 & -812.535346 & 0.000497 & 0.311872 & $10.93 \%$ \\
\hline 1aP & -812.487538 & -812.468143 & -812.467199 & -812.533936 & 0.001907 & 1.196661 & $2.45 \%$ \\
\hline $1 \mathrm{aQ}$ & -812.485637 & -812.466483 & -812.465539 & -812.532540 & 0.003303 & 2.072664 & $0.56 \%$ \\
\hline 1aR & -812.489014 & -812.469888 & -812.468944 & -812.534699 & 0.001144 & 0.717871 & $5.50 \%$ \\
\hline
\end{tabular}

$E, E^{\prime}, H, G$ : total energy, total energy with zero point energy (ZPE), enthalpy, and Gibbs free energy

Figure S2. Main conformers of 1a.

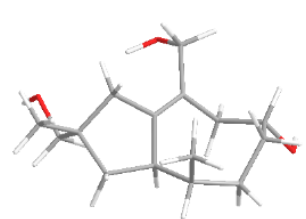

$1 a A$

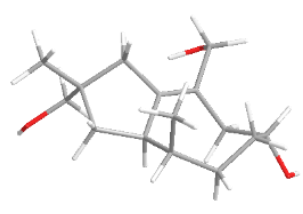

$1 a F$

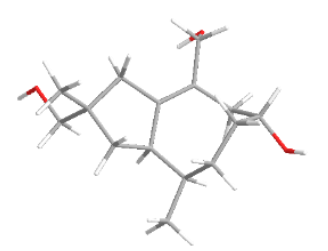

1aK

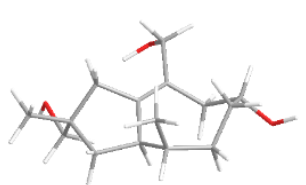

$1 a B$

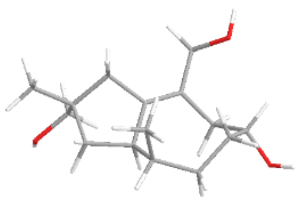

$1 a G$

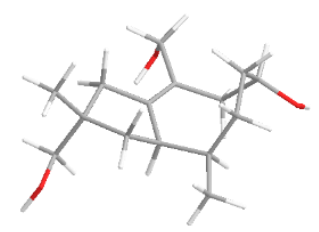

1aL

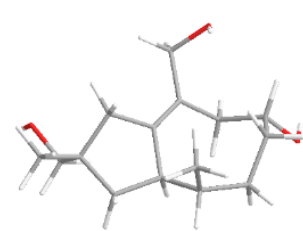

1aC

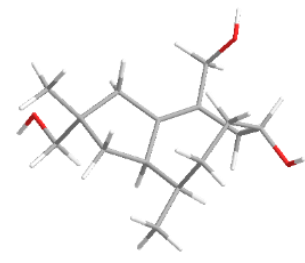

1aH

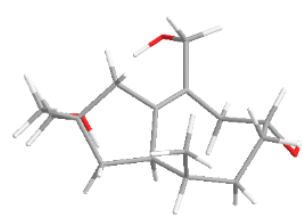

1aM

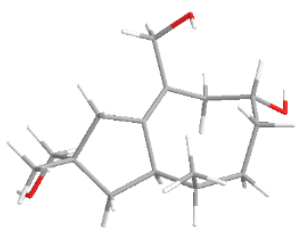

1aD

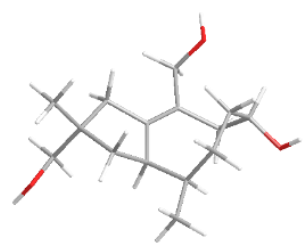

1 aI

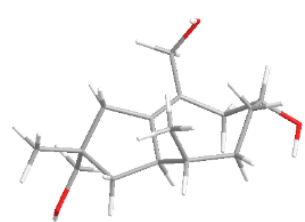

$1 a N$

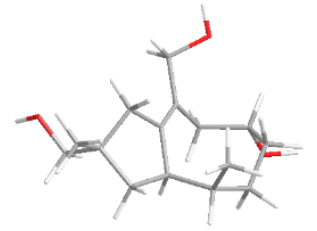

1aE

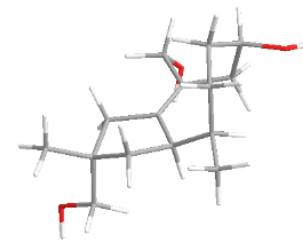

1aJ

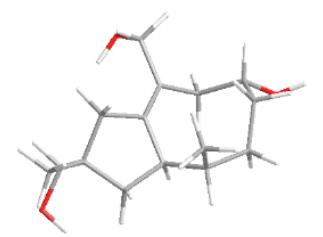

$1 \mathrm{aO}$ 


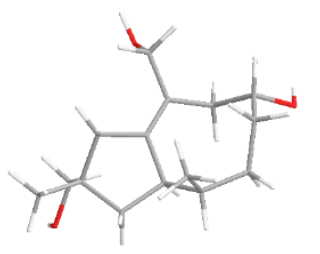

$1 \mathrm{aP}$

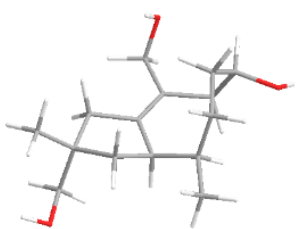

$1 \mathrm{aQ}$

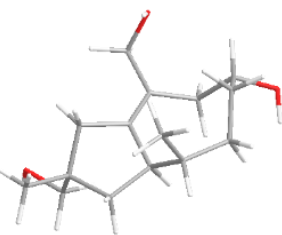

1aR

Table S2. Energy analysis for conformers of $\mathbf{1 b A - 1 b Y}$ at B3LYP/6-31G(d) level in the gas phase

\begin{tabular}{|c|c|c|c|c|c|c|c|}
\hline Species & $E^{\prime}=E+Z P E$ & $E$ & $H$ & $G$ & $\Delta G$ & $\Delta E(\mathrm{kcal} / \mathrm{mol})$ & PE\% \\
\hline $\mathbf{1 b A}$ & -812.489120 & -812.469944 & -812.469000 & -812.535037 & 0.000729 & 0.457454 & $7.44 \%$ \\
\hline $1 \mathrm{bB}$ & -812.488795 & -812.469728 & -812.468783 & -812.534377 & 0.001389 & 0.871611 & $3.70 \%$ \\
\hline $1 \mathrm{bC}$ & -812.489439 & -812.470307 & -812.469363 & -812.535164 & 0.000602 & 0.377761 & $8.51 \%$ \\
\hline 1bD & -812.484554 & -812.465349 & -812.464405 & -812.531126 & 0.004640 & 2.911644 & $0.12 \%$ \\
\hline $1 \mathrm{bE}$ & -812.489744 & -812.470482 & -812.469537 & -812.535766 & 0 & 0 & $16.11 \%$ \\
\hline $1 \mathrm{bF}$ & -812.488843 & -812.469629 & -812.468684 & -812.534879 & 0.000887 & 0.556601 & $6.29 \%$ \\
\hline $1 \mathrm{bG}$ & -812.488980 & -812.469763 & -812.468818 & -812.535014 & 0.000752 & 0.471887 & $7.26 \%$ \\
\hline 1bH & -812.485044 & -812.465798 & -812.464854 & -812.531660 & 0.004106 & 2.576554 & $0.21 \%$ \\
\hline 1bI & -812.484450 & -812.465228 & -812.464284 & -812.531294 & 0.004472 & 2.806222 & $0.14 \%$ \\
\hline 1bJ & -812.489694 & -812.470607 & -812.469663 & -812.535525 & 0.000241 & 0.151230 & $12.48 \%$ \\
\hline 1bK & -812.488684 & -812.469485 & -812.468541 & -812.534634 & 0.001132 & 0.710341 & $4.85 \%$ \\
\hline $1 \mathrm{bL}$ & -812.484147 & -812.464819 & -812.463875 & -812.530847 & 0.004919 & 3.086719 & $0.09 \%$ \\
\hline $1 \mathrm{bM}$ & -812.487101 & -812.467786 & -812.466842 & -812.533262 & 0.002504 & 1.571284 & $1.13 \%$ \\
\hline $1 b N$ & -812.489444 & -812.470104 & -812.469160 & -812.535624 & 0.000142 & 0.089106 & $13.86 \%$ \\
\hline $1 \mathrm{bO}$ & -812.488508 & -812.469294 & -812.468350 & -812.534449 & 0.001317 & 0.826430 & $3.99 \%$ \\
\hline $1 b P$ & -812.487721 & -812.468427 & -812.467483 & -812.533758 & 0.002008 & 1.260039 & $1.92 \%$ \\
\hline 1bQ & -812.484202 & -812.464981 & -812.464036 & -812.530688 & 0.005078 & 3.186493 & $0.07 \%$ \\
\hline 1bR & -812.483852 & -812.464545 & -812.463601 & -812.530324 & 0.005442 & 3.414907 & $0.05 \%$ \\
\hline $1 \mathrm{bS}$ & -812.484041 & -812.464735 & -812.463791 & -812.530740 & 0.005026 & 3.153863 & $0.08 \%$ \\
\hline $1 \mathrm{bT}$ & -812.487969 & -812.468778 & -812.467833 & -812.533919 & 0.001847 & 1.159010 & $2.28 \%$ \\
\hline $1 \mathrm{bU}$ & -812.489132 & -812.469963 & -812.469019 & -812.534889 & 0.000877 & 0.550326 & $6.36 \%$ \\
\hline $1 \mathrm{bV}$ & -812.488476 & -812.469383 & -812.468439 & -812.534101 & 0.001665 & 1.044803 & $2.76 \%$ \\
\hline $1 b W$ & -812.484545 & -812.465199 & -812.464255 & -812.531331 & 0.004435 & 2.783005 & $0.15 \%$ \\
\hline $1 b X$ & -812.484607 & -812.465395 & -812.464451 & -812.530947 & 0.004819 & 3.023968 & $0.10 \%$ \\
\hline $1 b Y$ & -812.484047 & -812.464852 & -812.463907 & -812.530483 & 0.005283 & 3.315133 & $0.06 \%$ \\
\hline
\end{tabular}

Figure S3. Main conformers of $\mathbf{1 b}$.

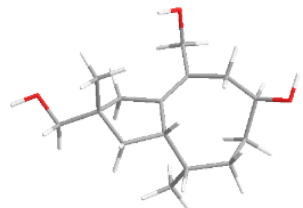

$1 \mathrm{bA}$

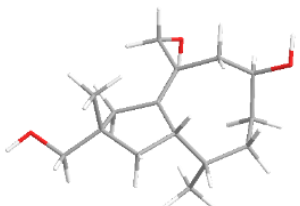

1bB

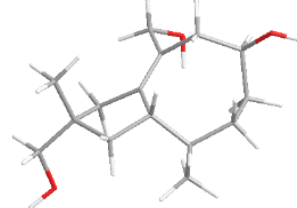

$1 \mathrm{bC}$

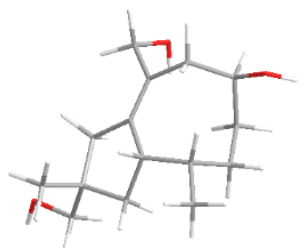

1bD

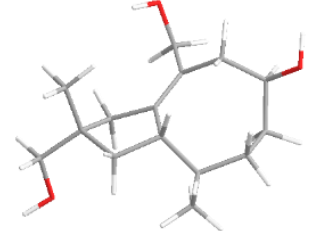

$1 \mathrm{bE}$ 


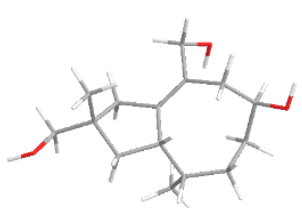

$1 b F$

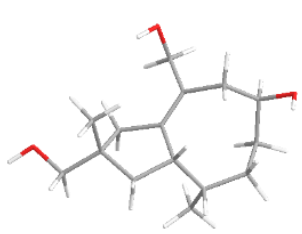

1bK

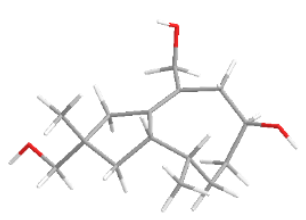

1bL

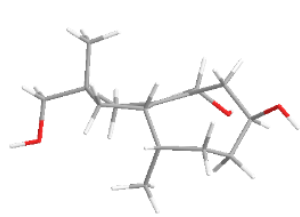

1bP

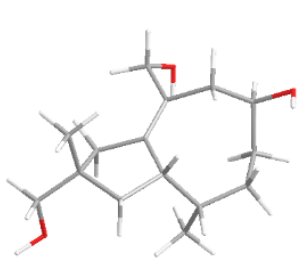

1bU

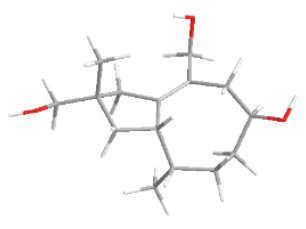

1bG
1bV

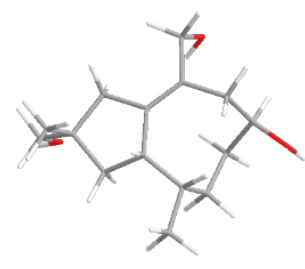

1bH

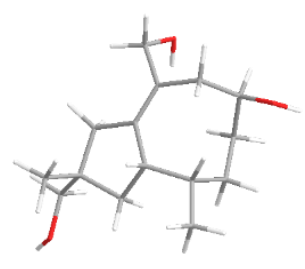

$1 b I$

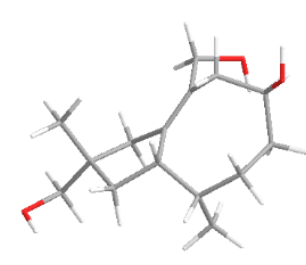

1bJ

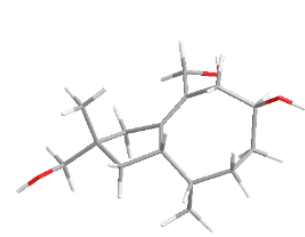

1bM

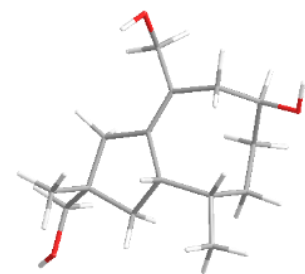

1bR

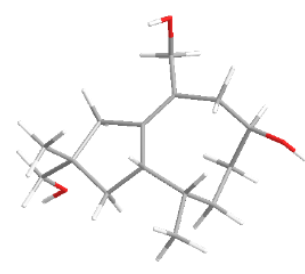

1bW

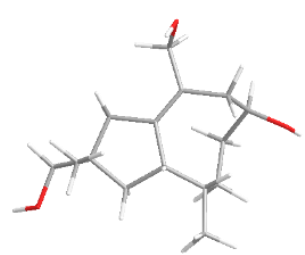

$1 b S$

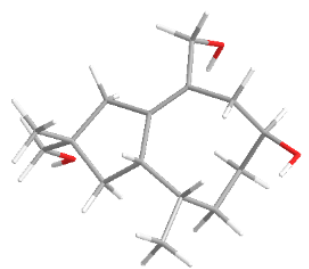

1bX

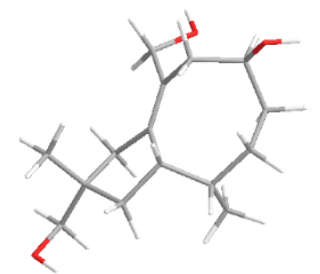

1bT

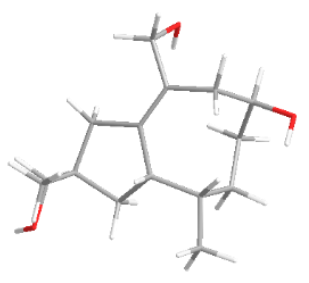

1bY 


\section{S1.2. Computational details for compound 2 (ECD)}

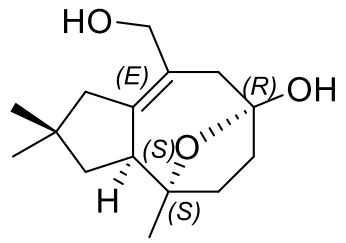

$2 a$

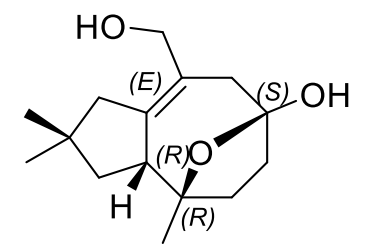

2b

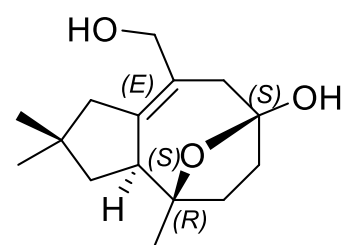

2c

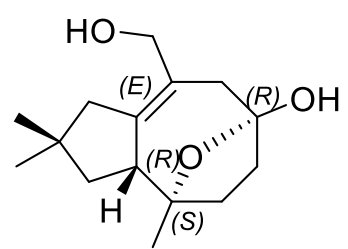

2d

Conformation search based on molecular mechanics with MMFF94S force fields were performed for $\mathbf{2 a - 2 d}$ gave 8, 9, 9, 9 stable conformers, respectively. All these conformers were further optimized on the B3LYP/6-31G(d) level of density functional theory method by Gaussian 16 program package. The ECD were calculated using B3LYP/6-31+G(d) level of theory with IEFPCM solvent model $(\mathrm{MeOH})$. The calculated ECD curves and weighted ECD were all generated using SpecDis 1.71 with $\sigma=0.25 \mathrm{eV}$, and UV shift $5 \mathrm{~nm}$, respectively.

Figure S4. Comparison of the calculated ECD spectra for $\mathbf{2 a - 2 d}$ with the experimental spectrum of $\mathbf{2}$ in methanol with PCM model.

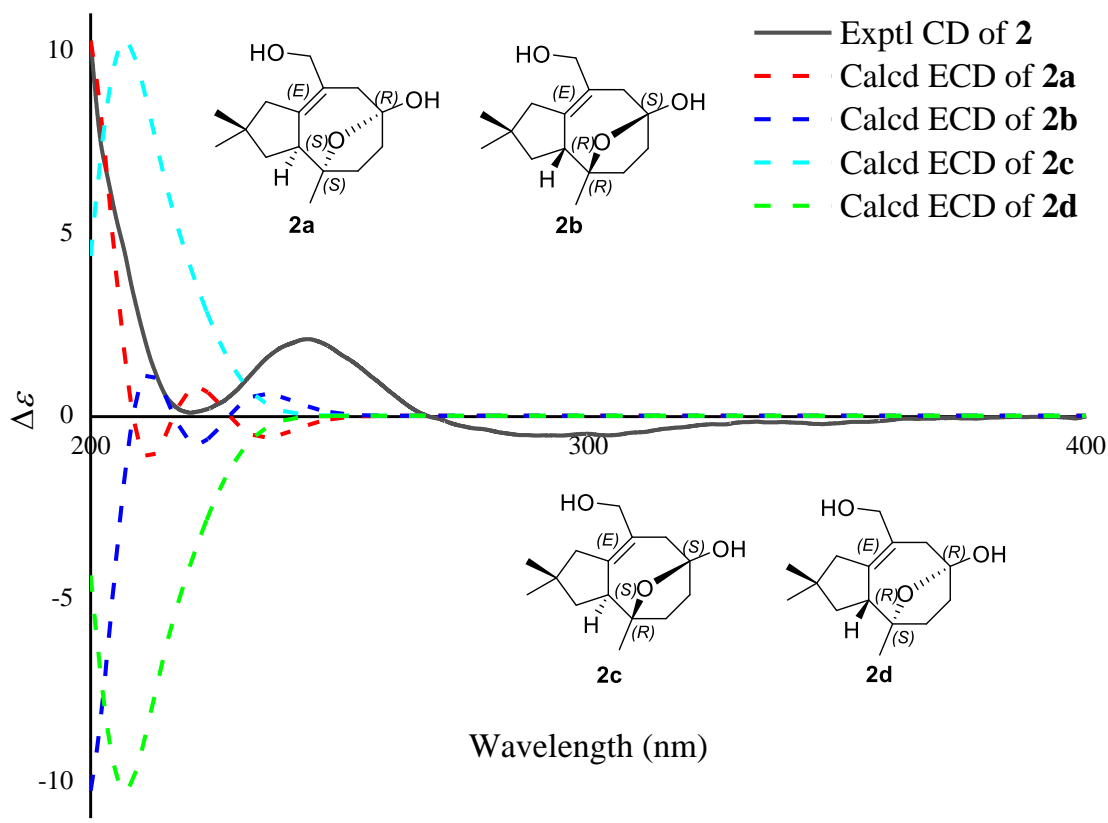

Table S3. Energy analysis for conformers of 2aA-2aH at B3LYP/6-31G(d) level in the gas phase

\begin{tabular}{lrrrrrrr}
\hline Species & $E^{\prime}=E+Z P E$ & $E$ & $H$ & $G$ & $\Delta G$ & $\Delta E(\mathrm{kcal} / \mathrm{mol})$ & $P E \%$ \\
\hline 2aA & -811.332924 & -811.315101 & -811.314157 & -811.376657 & 0.000955 & 0.599272 & $10.66 \%$ \\
2aB & -811.333318 & -811.315542 & -811.314598 & -811.377023 & 0.000589 & 0.369603 & $15.71 \%$ \\
$\mathbf{2 a C}$ & -811.333569 & -811.315763 & -811.314819 & -811.377247 & 0.000365 & 0.229041 & $19.91 \%$ \\
$\mathbf{2 a D}$ & -811.333975 & -811.316223 & -811.315278 & -811.377612 & 0 & 0 & $29.32 \%$
\end{tabular}




\begin{tabular}{lllllllr}
\hline $\mathbf{2 a E}$ & -811.331884 & -811.313870 & -811.312926 & -811.376092 & 0.001520 & 0.953814 & $5.86 \%$ \\
$\mathbf{2 a F}$ & -811.332578 & -811.314594 & -811.313649 & -811.376661 & 0.000951 & 0.596762 & $10.70 \%$ \\
$\mathbf{2 a G}$ & -811.331311 & -811.313370 & -811.312425 & -811.375217 & 0.002395 & 1.502885 & $2.32 \%$ \\
$\mathbf{2 a H}$ & -811.332316 & -811.314438 & -811.313494 & -811.376038 & 0.001574 & 0.987700 & $5.53 \%$ \\
\hline
\end{tabular}

Figure S5. Main conformers for $\mathbf{2 a}$.

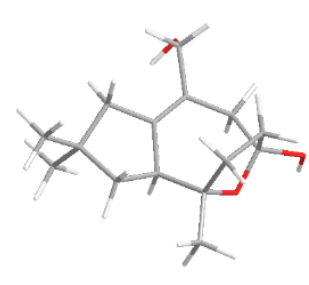

$\mathbf{2 a A}$

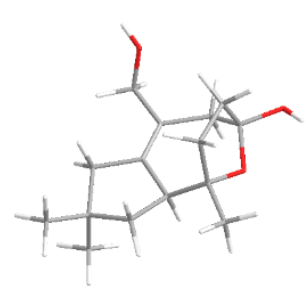

$2 \mathbf{a F}$

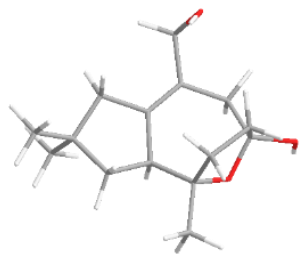

2aB

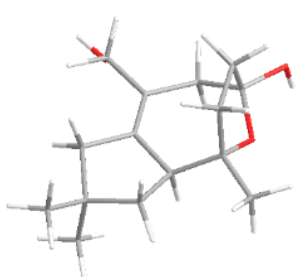

$2 \mathbf{a G}$

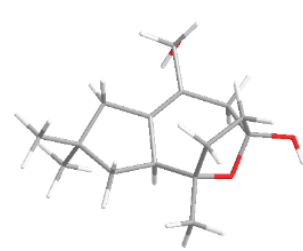

$2 \mathrm{aC}$

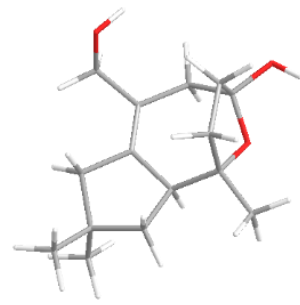

2aH

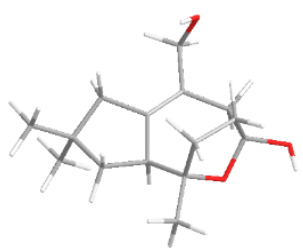

2aD

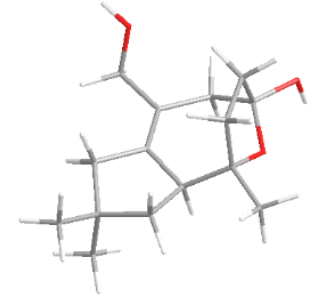

2aE

Table S4. Energy analysis for conformers of $\mathbf{2 b A - 2 b I}$ at B3LYP/6-31G(d) level in the gas phase

\begin{tabular}{lrrrrrrr}
\hline Species & $E^{\prime}=E+Z P E$ & $E$ & $H$ & $G$ & $\Delta G$ & $\Delta E(\mathrm{kcal} / \mathrm{mol})$ & $P E \%$ \\
\hline 2bA & -811.332924 & -811.315101 & -811.314157 & -811.376657 & 0.000955 & 0.599272 & $10.40 \%$ \\
2bB & -811.333318 & -811.315542 & -811.314598 & -811.377023 & 0.000589 & 0.369603 & $15.33 \%$ \\
$\mathbf{2 b C}$ & -811.333569 & -811.315763 & -811.314819 & -811.377247 & 0.000365 & 0.229041 & $19.44 \%$ \\
$\mathbf{2 b D}$ & -811.333975 & -811.316223 & -811.315278 & -811.377612 & 0 & 0 & $28.62 \%$ \\
$\mathbf{2 b E}$ & -811.331884 & -811.313870 & -811.312926 & -811.376092 & 0.001520 & 0.953814 & $5.72 \%$ \\
$\mathbf{2 b F}$ & -811.332578 & -811.314594 & -811.313649 & -811.376661 & 0.000951 & 0.596762 & $10.45 \%$ \\
$\mathbf{2 b G}$ & -811.331311 & -811.313370 & -811.312425 & -811.375217 & 0.002395 & 1.502885 & $2.26 \%$ \\
$\mathbf{2 b H}$ & -811.331495 & -811.313611 & -811.312667 & -811.375264 & 0.002348 & 1.473392 & $2.38 \%$ \\
$\mathbf{2 b I}$ & -811.332316 & -811.314438 & -811.313494 & -811.376038 & 0.001574 & 0.987700 & $5.40 \%$ \\
\hline
\end{tabular}

Figure S6. Main conformers for $\mathbf{2 b}$.

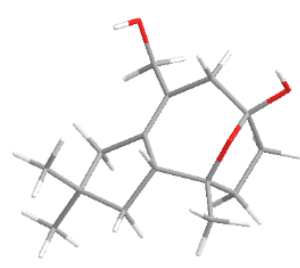

2bA

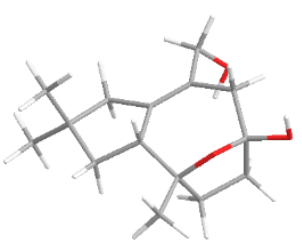

2bB

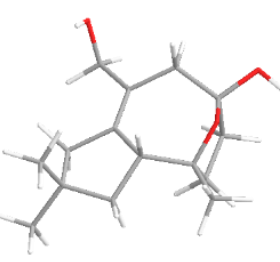

2bC

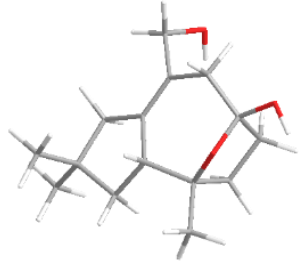

2bD

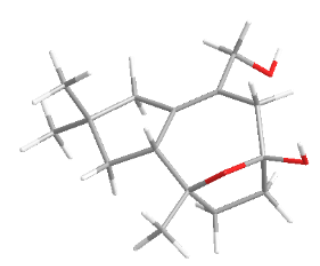

2bE 


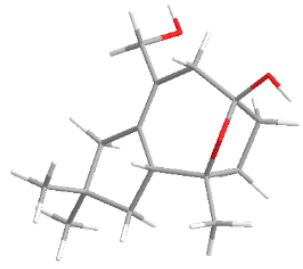

$2 \mathbf{b F}$

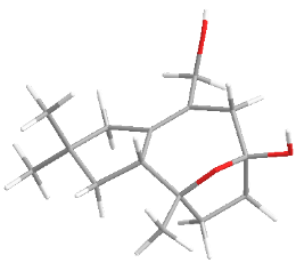

$2 \mathrm{bG}$

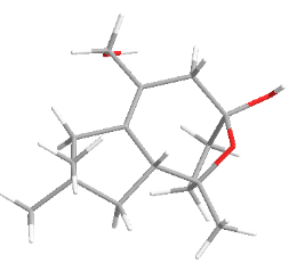

$\mathbf{2 b H}$

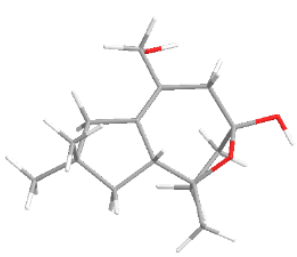

$2 \mathbf{b I}$

Table S5. Energy analysis for conformers of 2cA-2cI at B3LYP/6-31G(d) level in the gas phase

\begin{tabular}{lrrrrrrr}
\hline Species & $E^{\prime}=E+Z P E$ & $E$ & $H$ & $G$ & $\Delta G$ & $\Delta E(\mathrm{kcal} / \mathrm{mol})$ & $P E \%$ \\
\hline $\mathbf{2 c A}$ & -811.321850 & -811.304207 & -811.303263 & -811.365862 & 0.000943 & 0.591741 & $7.74 \%$ \\
$\mathbf{2 c B}$ & -811.322064 & -811.304302 & -811.303358 & -811.366462 & 0.000343 & 0.215236 & $14.62 \%$ \\
$\mathbf{2 c C}$ & -811.321786 & -811.304024 & -811.303080 & -811.366156 & 0.000649 & 0.407254 & $10.57 \%$ \\
$\mathbf{2 c D}$ & -811.322172 & -811.304508 & -811.303564 & -811.366204 & 0.000601 & 0.377133 & $11.13 \%$ \\
$\mathbf{2 c E}$ & -811.321494 & -811.303688 & -811.302744 & -811.365915 & 0.000890 & 0.558483 & $8.19 \%$ \\
$\mathbf{2 c F}$ & -811.320198 & -811.302288 & -811.301344 & -811.364952 & 0.001853 & 1.162775 & $2.95 \%$ \\
$\mathbf{2 c G}$ & -811.320513 & -811.302621 & -811.301677 & -811.365429 & 0.001376 & 0.863453 & $4.89 \%$ \\
$\mathbf{2 c H}$ & -811.322522 & -811.304785 & -811.303841 & -811.366805 & 0 & 0 & $21.04 \%$ \\
$\mathbf{2 c I}$ & -811.322384 & -811.304649 & -811.303704 & -811.366702 & 0.000103 & 0.064633 & $18.86 \%$ \\
\hline
\end{tabular}

$E, E^{\prime}, H, G$ : total energy, total energy with zero point energy (ZPE), enthalpy, and Gibbs free energy

Figure S7. Main conformers of 2c.

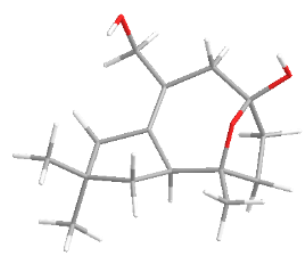

$2 \mathbf{c A}$

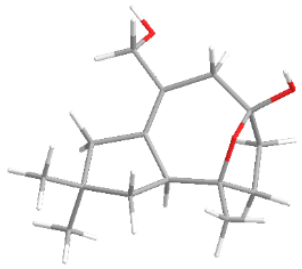

$2 \mathrm{cF}$

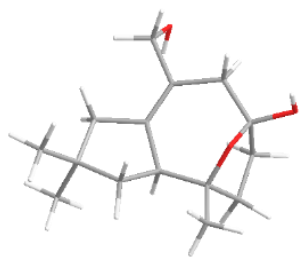

$2 \mathbf{c B}$

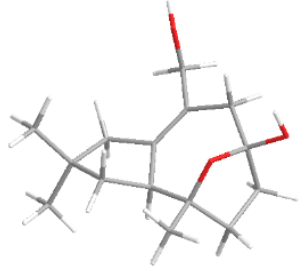

$2 \mathrm{cG}$

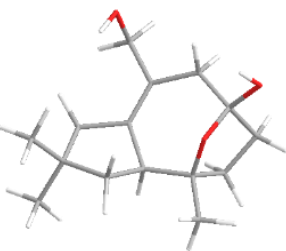

$2 \mathrm{cC}$

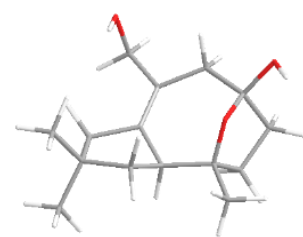

$2 \mathrm{cH}$

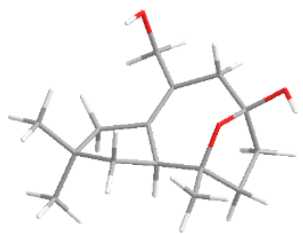

$2 \mathrm{cD}$

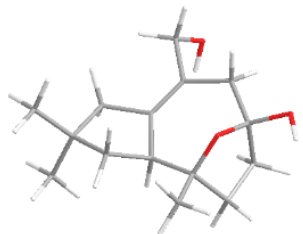

$2 \mathbf{c I}$

Table S6. Energy analysis for conformers of 2dA-2dI at B3LYP/6-31G(d) level in the gas phase

\begin{tabular}{lrrrrrrr}
\hline Species & $E^{\prime}=E+Z P E$ & $E$ & $H$ & $G$ & $\Delta G$ & $\Delta E(\mathrm{kcal} / \mathrm{mol})$ & $P E \%$ \\
\hline $\mathbf{2 d A}$ & -811.321850 & -811.304207 & -811.303263 & -811.365862 & 0.000943 & 0.591741 & $7.75 \%$ \\
$\mathbf{2 d B}$ & -811.322064 & -811.304302 & -811.303358 & -811.366462 & 0.000343 & 0.215236 & $14.64 \%$ \\
$\mathbf{2 d C}$ & -811.321786 & -811.304024 & -811.303080 & -811.366156 & 0.000649 & 0.407254 & $10.58 \%$
\end{tabular}




\begin{tabular}{lrrrrrrr}
\hline $\mathbf{2 d D}$ & -811.322172 & -811.304508 & -811.303564 & -811.366204 & 0.000601 & 0.377133 & $11.14 \%$ \\
$\mathbf{2 d E}$ & -811.321494 & -811.303688 & -811.302744 & -811.365915 & 0.000890 & 0.558483 & $8.20 \%$ \\
$\mathbf{2 d F}$ & -811.320198 & -811.302288 & -811.301344 & -811.364952 & 0.001853 & 1.162775 & $2.95 \%$ \\
$\mathbf{2 d G}$ & -811.320513 & -811.302621 & -811.301676 & -811.365430 & 0.001375 & 0.862826 & $4.90 \%$ \\
$\mathbf{2 d H}$ & -811.322522 & -811.304785 & -811.303841 & -811.366805 & 0 & 0 & $21.06 \%$ \\
$\mathbf{2 d I}$ & -811.322383 & -811.304649 & -811.303705 & -811.366697 & 0.000108 & 0.067771 & $18.78 \%$ \\
\hline
\end{tabular}

Figure S8. Main conformers for $\mathbf{2 d}$.

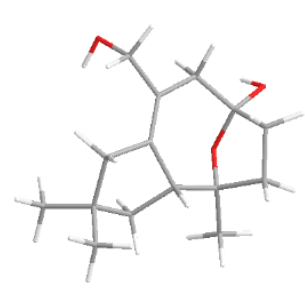

2dA

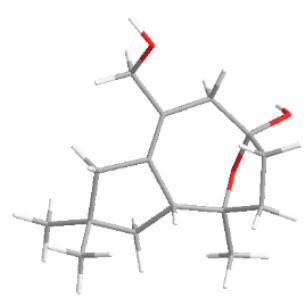

2dF

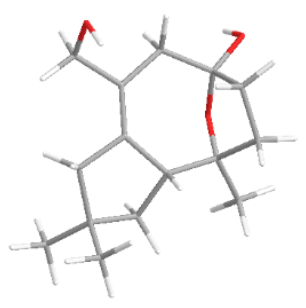

2dB

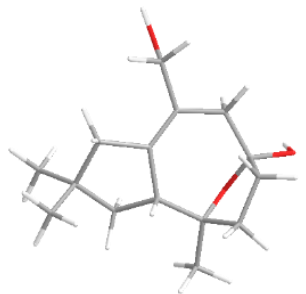

2dG

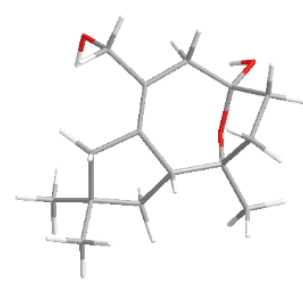

2dC

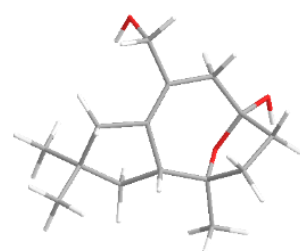

2dH

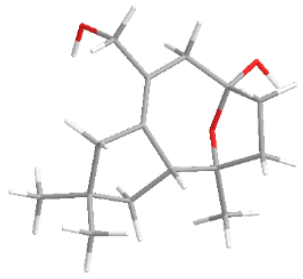

2dD

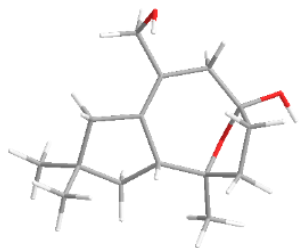

2dI

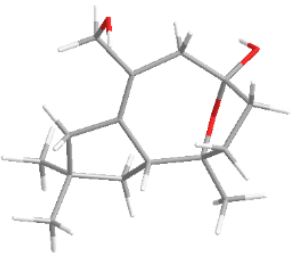

2dE 


\section{S1.3. Computational details for compound 3 (ECD)}

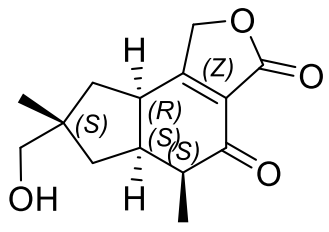

$3 a$

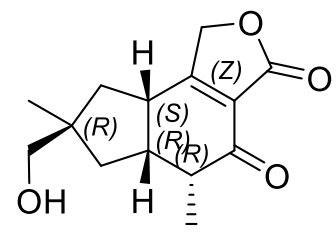

3b

Conformation search based on molecular mechanics with MMFF94S force fields were performed for $\mathbf{3 a}$ and $\mathbf{3 b}$ gave 6 and 6 stable conformers, respectively. All these conformers were further optimized by the density functional theory method at the B3LYP/6-31G(d) level by Gaussian 16 program package. The ECD were calculated using B3LYP/6-31+G(d) level in methanol with PCM model on B3LYP/6-31+G(d). The calculated ECD curves and weighted ECD were all generated using SpecDis 1.71 with $\sigma=0.25 \mathrm{eV}$, and UV shift $-11 \mathrm{~nm}$, respectively.

Figure S9. Comparison of the calculated ECD spectra for $\mathbf{3 a}$ and $\mathbf{3 b}$ with the experimental spectrum of $\mathbf{3}$ in methanol with PCM model.

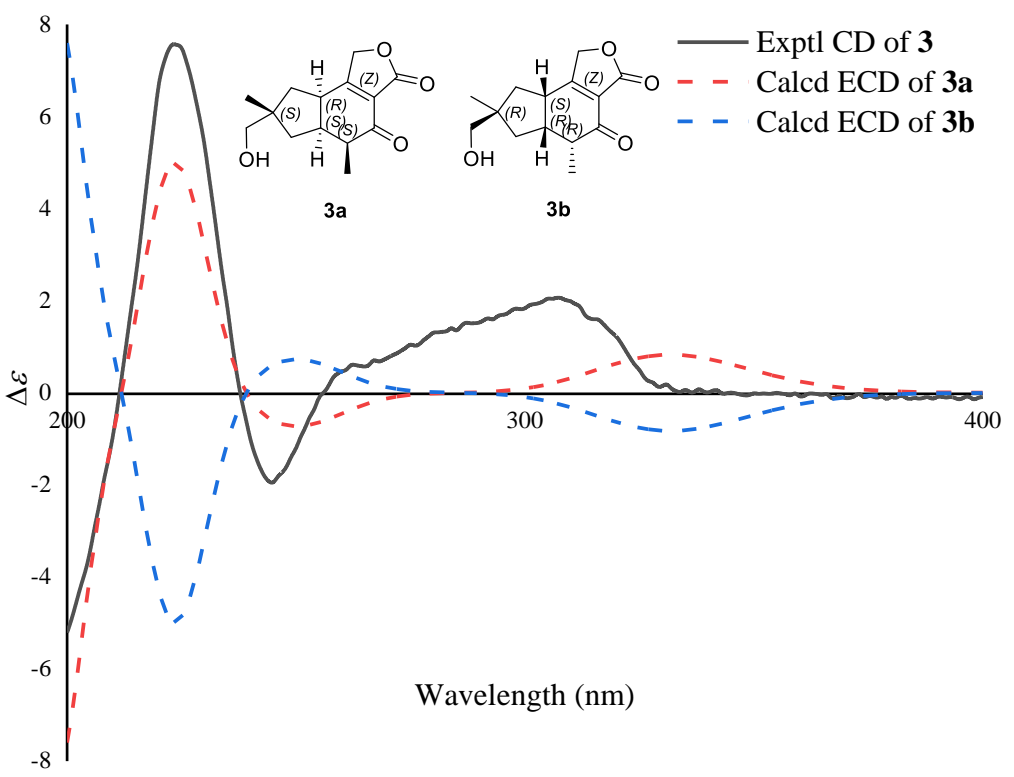

Table S7. Energy analysis for conformers of 3aA-3aF at B3LYP/6-31G(d) level in the gas phase

\begin{tabular}{lrrrrrrr}
\hline Species & $E^{\prime}=E+Z P E$ & $E$ & $H$ & $G$ & $\Delta G$ & $\Delta E(\mathrm{kcal} / \mathrm{mol})$ & $P E \%$ \\
\hline 3aA & -844.930037 & -844.913368 & -844.912424 & -844.973928 & 0.000623 & 0.390938 & $15.09 \%$ \\
3aB & -844.929330 & -844.912629 & -844.911684 & -844.973396 & 0.001155 & 0.724773 & $8.59 \%$ \\
$\mathbf{3 a C}$ & -844.930037 & -844.913368 & -844.912424 & -844.973927 & 0.000624 & 0.391566 & $15.07 \%$ \\
$\mathbf{3 a D}$ & -844.930184 & -844.913685 & -844.912741 & -844.973839 & 0.000712 & 0.446787 & $13.73 \%$ \\
$\mathbf{3 a E}$ & -844.929854 & -844.913253 & -844.912309 & -844.974111 & 0.000440 & 0.276104 & $18.32 \%$ \\
$\mathbf{3 a F}$ & -844.929511 & -844.912763 & -844.911819 & -844.974551 & 0 & 0 & $29.20 \%$ \\
\hline
\end{tabular}


$E, E^{\prime}, H, G$ : total energy, total energy with zero point energy $(Z P E)$, enthalpy, and Gibbs free energy

Figure S10. Main conformers for 3a.

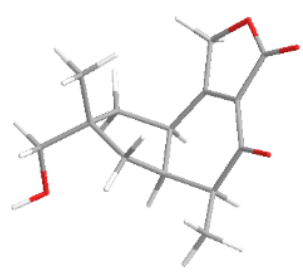

$3 \mathbf{a A}$

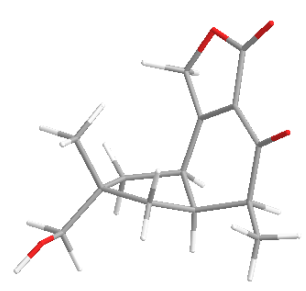

3aF

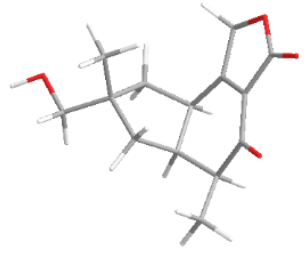

3aB

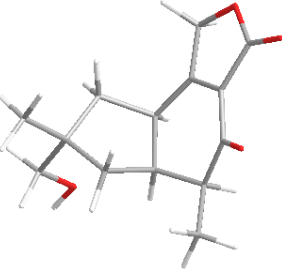

$3 \mathrm{aC}$

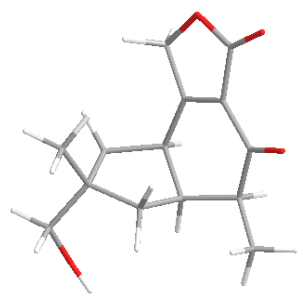

3aD

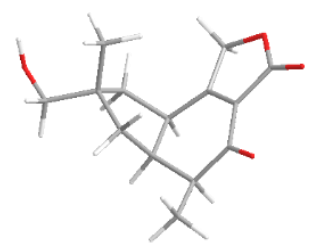

3aE

Table S8. Energy analysis for conformers of 3bA-3bF at B3LYP/6-31G(d) level in the gas phase

\begin{tabular}{lrrrrrrr}
\hline Species & $E^{\prime}=E+Z P E$ & $E$ & $H$ & $G$ & $\Delta G$ & $\Delta E(\mathrm{kcal} / \mathrm{mol})$ & $P E \%$ \\
\hline 3bA & -844.930037 & -844.913368 & -844.912424 & -844.973928 & 0.000623 & 0.390938 & $15.11 \%$ \\
3bB & -844.929330 & -844.912629 & -844.911684 & -844.973396 & 0.001155 & 0.724773 & $8.60 \%$ \\
3bC & -844.930037 & -844.913368 & -844.912424 & -844.973927 & 0.000624 & 0.391566 & $15.09 \%$ \\
3bD & -844.930184 & -844.913685 & -844.912741 & -844.973839 & 0.000712 & 0.446787 & $13.75 \%$ \\
3bE & -844.929853 & -844.913253 & -844.912309 & -844.974105 & 0.000446 & 0.279869 & $18.22 \%$ \\
3bF & -844.929511 & -844.912763 & -844.911819 & -844.974551 & 0 & 0 & $29.24 \%$ \\
\hline
\end{tabular}

Figure S11. Main conformers for $\mathbf{3 b .}$

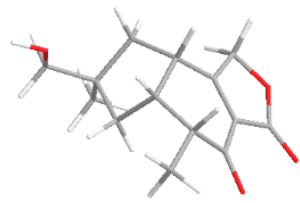

3bA

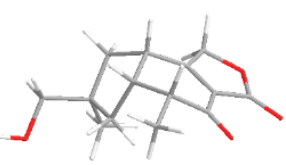

3bF

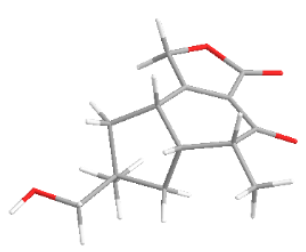

$3 \mathbf{b B}$

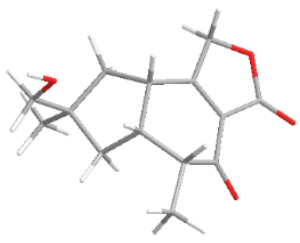

3bC

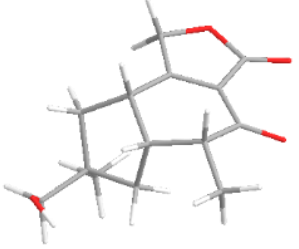

3bD

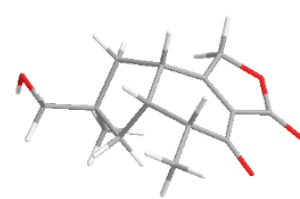

$3 \mathbf{b E}$

S1.4. Computational details for compound 4 (ECD) 


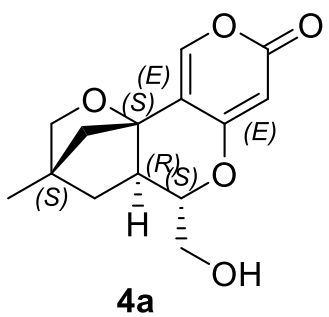

$4 a$

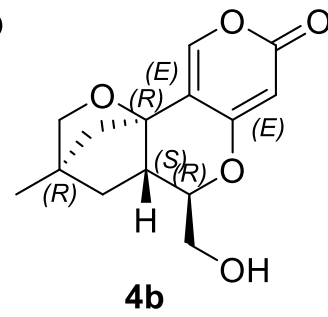

Conformation search based on molecular mechanics with MMFF94S force fields were performed for $\mathbf{4 a}$ and $\mathbf{4 b}$ gave 3 and 3 stable conformers, respectively. All these conformers were further optimized by the density functional theory method at the B3LYP/6-31G(d) level by Gaussian 16 program package. The ECD were calculated using B3LYP/6-31+G(d) level in methanol with PCM model on B3LYP/6-31+G(d). The calculated ECD curves and weighted ECD were all generated using SpecDis 1.71 with $\sigma=0.25 \mathrm{eV}$, and UV shift $6 \mathrm{~nm}$, respectively.

Figure S12. Comparison of the calculated ECD spectra for $\mathbf{4 a}$ and $\mathbf{4 b}$ with the experimental spectrum of 4 in methanol with PCM model.

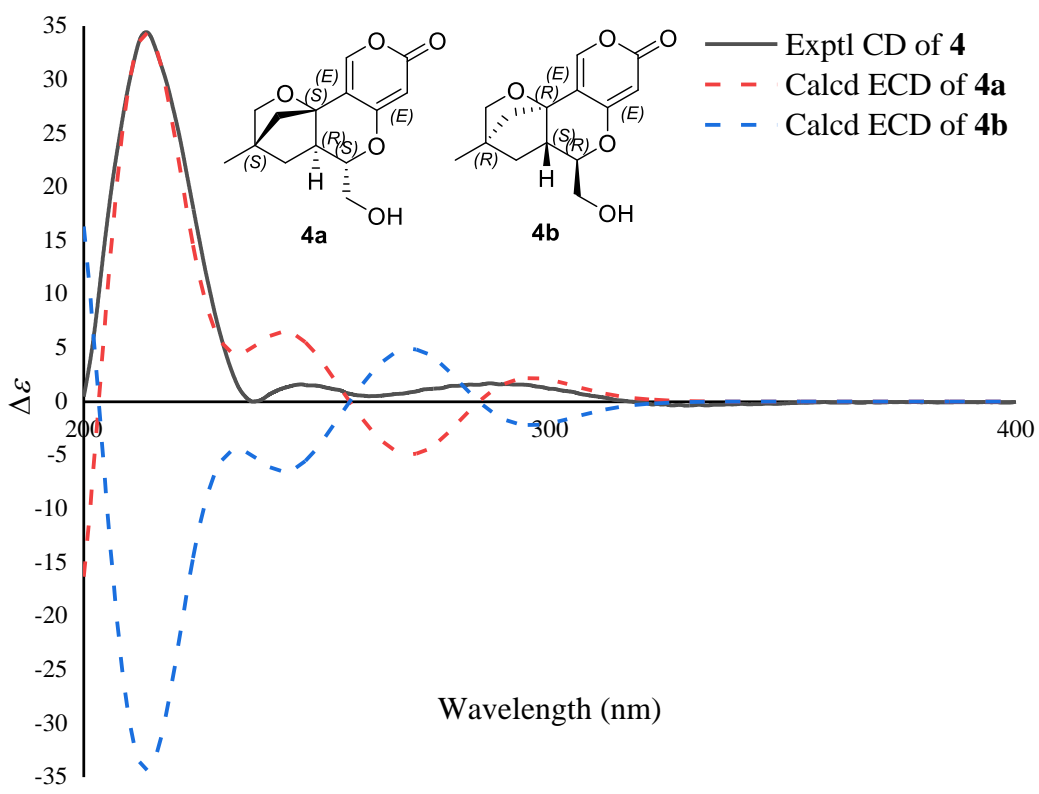

Table S9. Energy analysis for conformers of $\mathbf{4 a A}-\mathbf{4 a C}$ at B3LYP/6-31G(d) level in the gas phase

\begin{tabular}{lrrrrrrr}
\hline Species & $E^{\prime}=E+Z P E$ & $E$ & $H$ & $G$ & $\Delta G$ & $\Delta E(\mathrm{kcal} / \mathrm{mol})$ & $P E \%$ \\
\hline $\mathbf{4 a A}$ & -918.934499 & -918.918717 & -918.917773 & -918.976998 & 0.000046 & 0.028865 & $47.95 \%$ \\
$\mathbf{4 a B}$ & -918.934696 & -918.918996 & -918.918052 & -918.977044 & 0 & 0 & $50.34 \%$ \\
$\mathbf{4 a C}$ & -918.930790 & -918.914746 & -918.913802 & -918.973855 & 0.003189 & 2.001128 & $1.71 \%$
\end{tabular}


Figure S13. Main conformers for $4 a$.

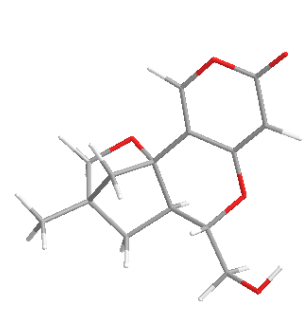

$4 a A$

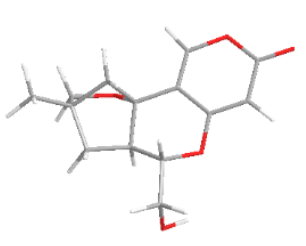

$4 a B$

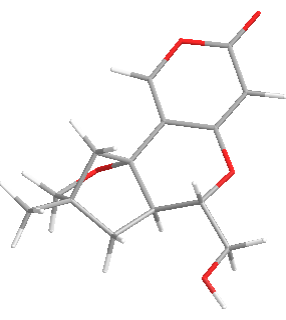

$4 a C$

Table S10. Energy analysis for conformers of $\mathbf{4 b A}-\mathbf{4 b C}$ at B3LYP/6-31G(d) level in the gas phase

\begin{tabular}{lrrrrrrr}
\hline Species & $E^{\prime}=E+Z P E$ & $E$ & $H$ & $G$ & $\Delta G$ & $\Delta E(\mathrm{kcal} / \mathrm{mol})$ & $P E \%$ \\
\hline 4bA & -918.934499 & -918.918717 & -918.917773 & -918.976998 & 0.000046 & 0.028865 & $47.95 \%$ \\
4bB & -918.934696 & -918.918996 & -918.918052 & -918.977044 & 0 & 0 & $50.34 \%$ \\
4bC & -918.930790 & -918.914746 & -918.913802 & -918.973855 & 0.003189 & 2.001128 & $1.71 \%$ \\
\hline
\end{tabular}

Figure S14. Main conformers for $4 b$.

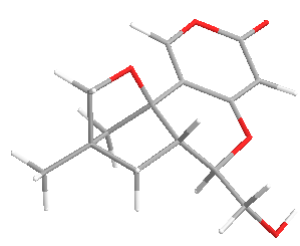

$4 \mathrm{bA}$

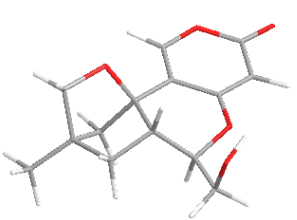

$4 \mathrm{bB}$

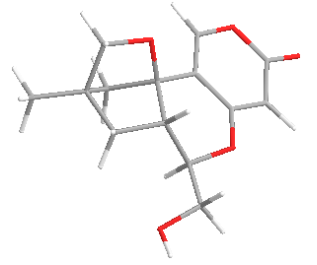

$4 b C$ 
Section S2. Immunosuppressive Tests of 1-4.

Table S11. Immunosuppressive data for 1-4.

\begin{tabular}{|c|c|c|c|c|c|}
\hline \multirow{2}{*}{ No. } & & \multicolumn{2}{|c|}{$\begin{array}{l}\text { ConA-induced T-cell } \\
\text { proliferation }\end{array}$} & \multicolumn{2}{|c|}{$\begin{array}{l}\text { LPS-induced B-cell } \\
\text { proliferation }\end{array}$} \\
\hline & $\begin{array}{l}\mathrm{CC}_{50} \\
(\mu \mathrm{M})\end{array}$ & $\begin{array}{l}\mathrm{IC}_{50} \\
(\mu \mathrm{M})\end{array}$ & $\mathrm{SI}^{\mathrm{a}}$ & $\begin{array}{c}\mathrm{IC}_{50} \\
(\mu \mathrm{M})\end{array}$ & $\mathrm{SI}^{\mathrm{a}}$ \\
\hline 1 & 219.3 & 78.3 & 2.8 & 121.1 & 1.8 \\
\hline 2 & 170.1 & $>200$ & $<0.8$ & 168.4 & 1.0 \\
\hline 3 & 76.3 & 12.3 & 6.2 & 127.4 & 0.6 \\
\hline 4 & 12.1 & 6.6 & 1.8 & 32.4 & 0.4 \\
\hline $\mathbf{C s}^{\mathrm{b}}{ }^{\mathrm{b}}$ & $>2.5$ & 0.03 & $>83.3$ & 0.32 & $>7.8$ \\
\hline
\end{tabular}

${ }^{\mathrm{a}} \mathrm{SI}$ (selectivity index) $=\mathrm{CC}_{50} / \mathrm{IC}_{50}$; ${ }^{\mathrm{b}}$ Positive control 
Section S3. NMR spectra for 1-4.

S3.1. NMR spectra (methanol- $d_{4}$ ) and HRESIMS for conosiligin A (1)

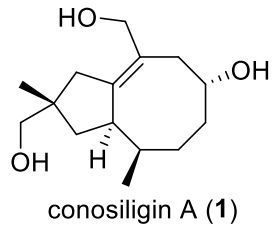

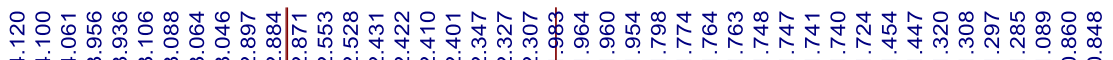

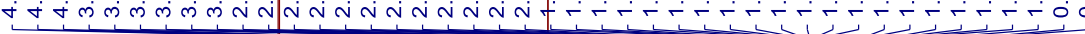

nosiligin A (1)

${ }^{1} \mathrm{H}$ NMR
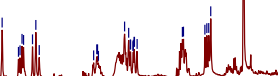

势管

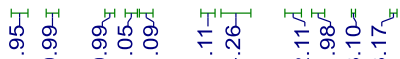
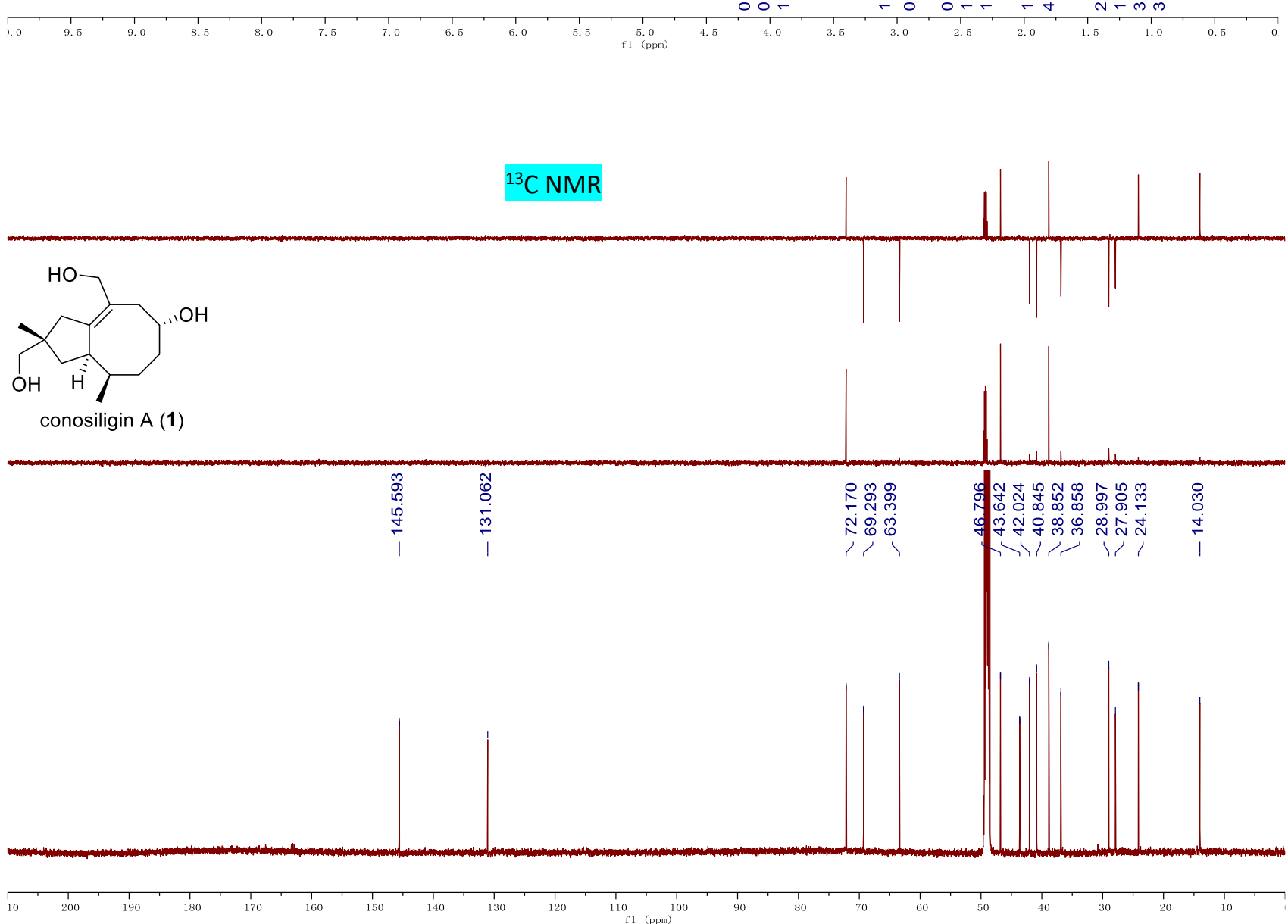

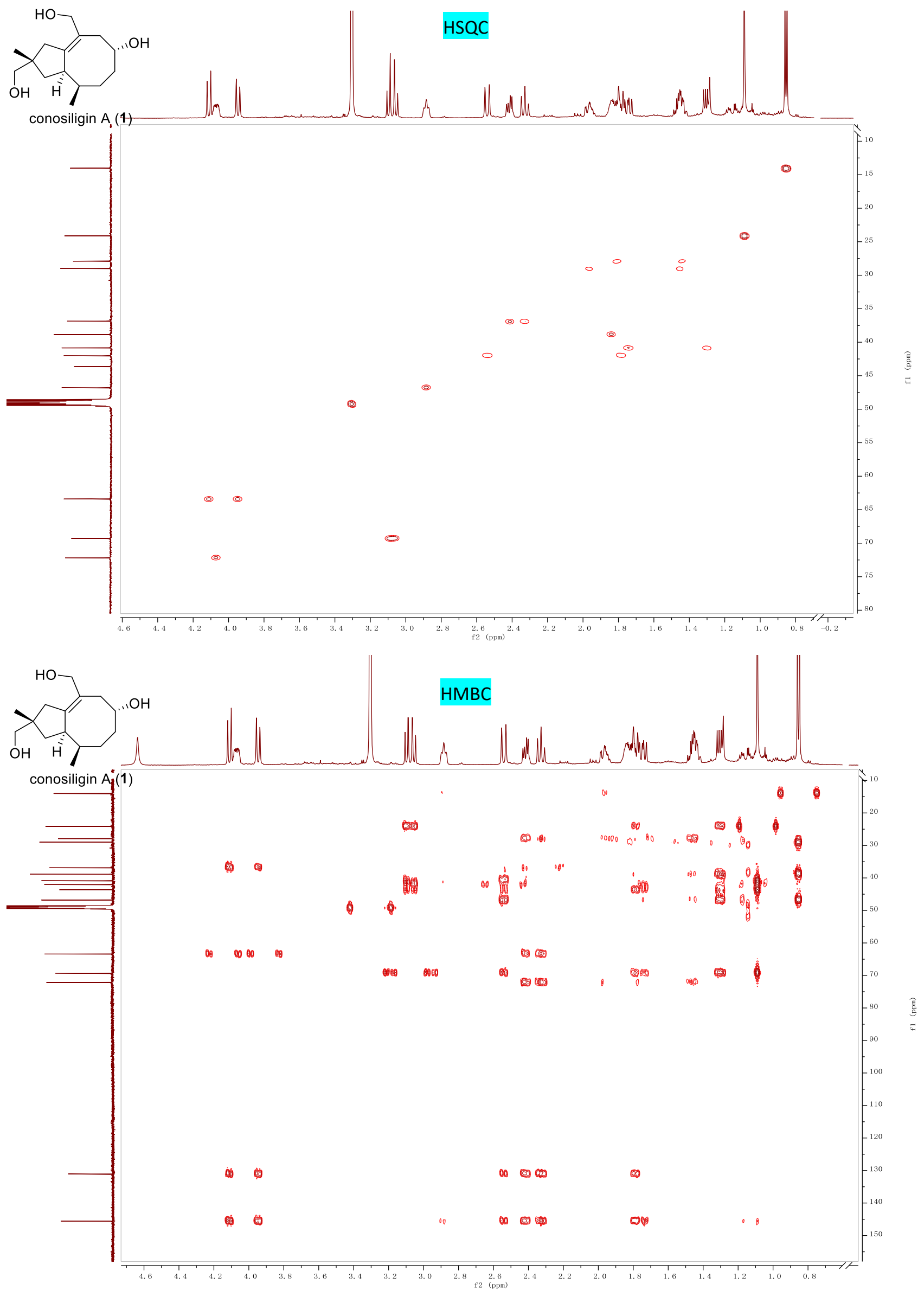

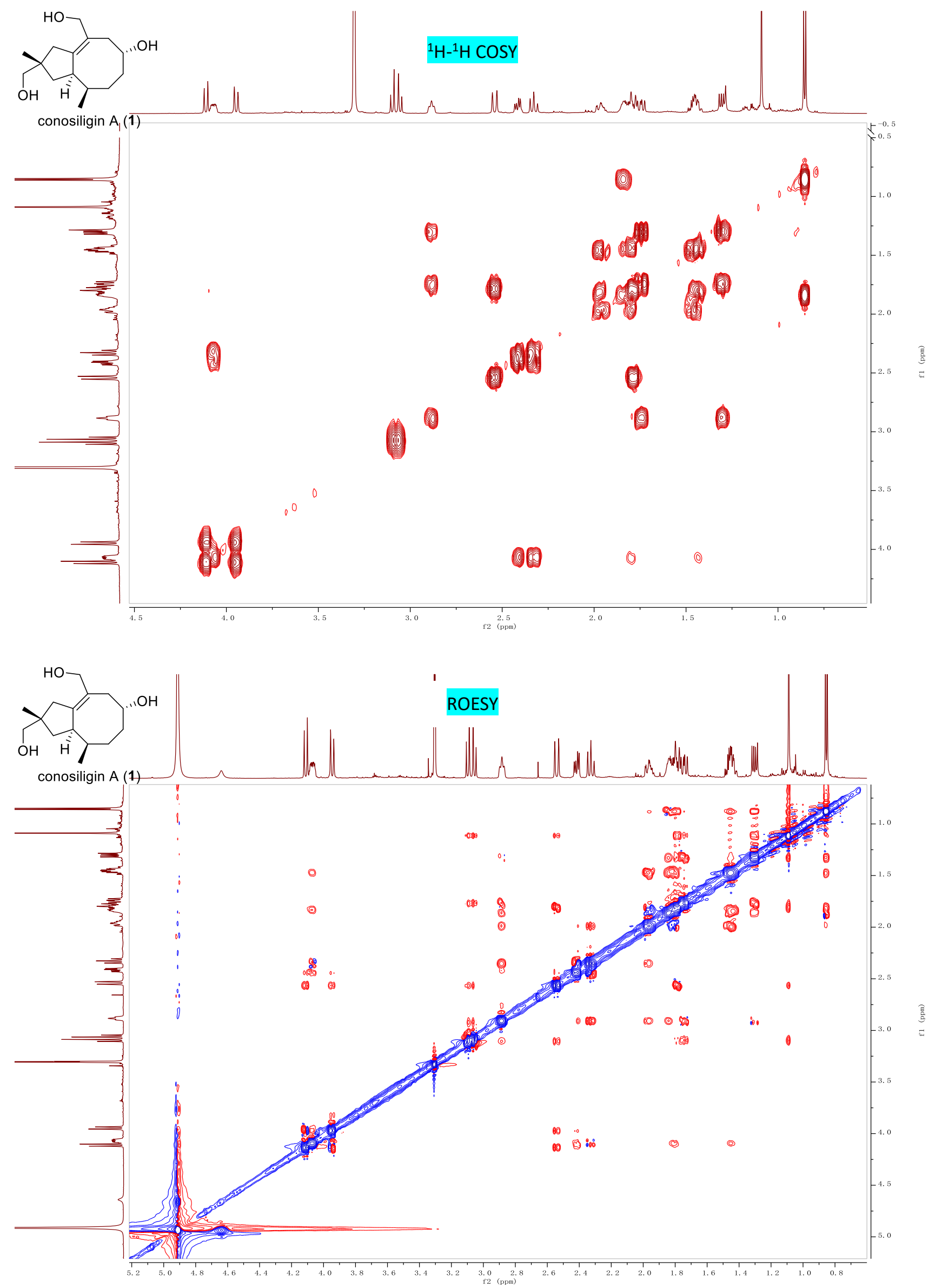


\section{HRESIMS}

Formula Predictor Report - LPCA-11.Icd

Data File: E:IDATAL2017/12201LPCA-11.Icd

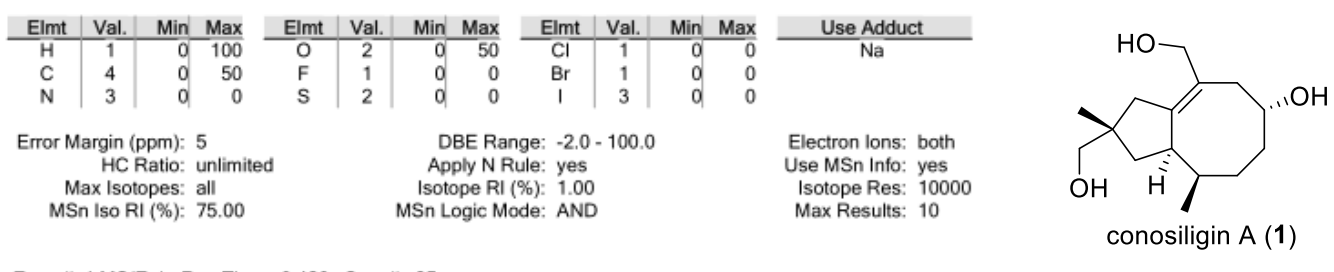

Event\#: $1 \mathrm{MS}(\mathrm{E}+)$ Ret. Time : 0.420 Scan\# : 85
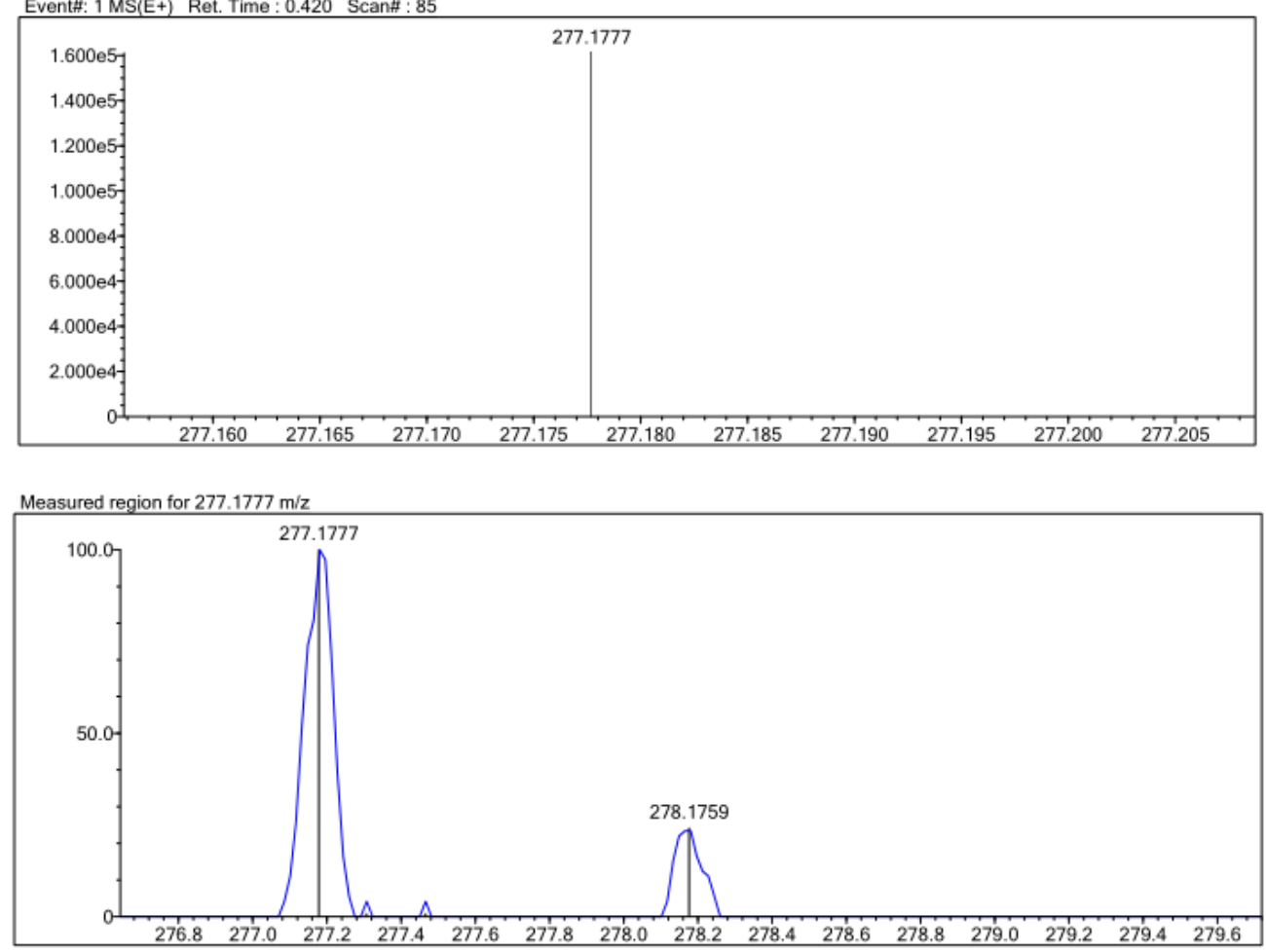

$\mathrm{C} 15 \mathrm{H} 26 \mathrm{O} 3[\mathrm{M}+\mathrm{Na}]+$ : Predicted region for $277.1774 \mathrm{~m} / \mathrm{z}$

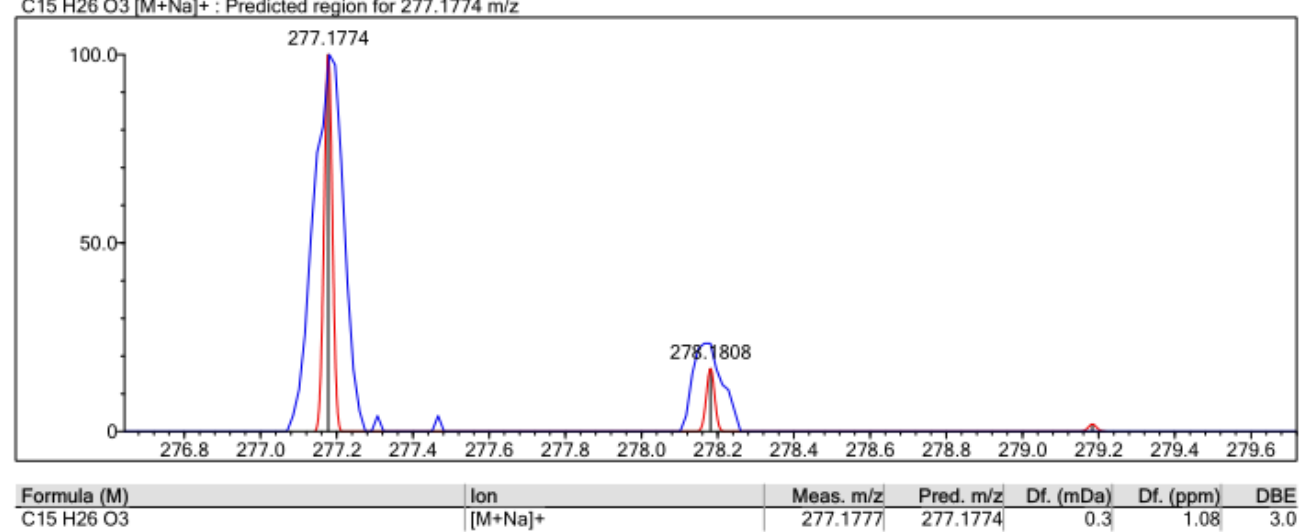


S3.2. NMR spectra ( $\left.\mathrm{CDCl}_{3}\right)$ and HRESIMS for conosiligin $B(2)$

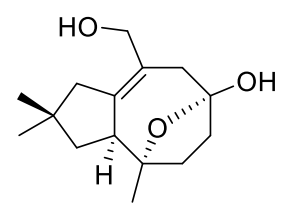

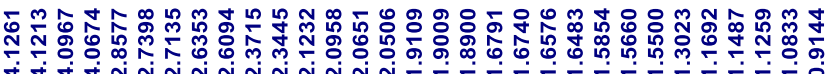

${ }^{1} \mathrm{H} N \mathrm{NMR}$

conosiligin B (2)

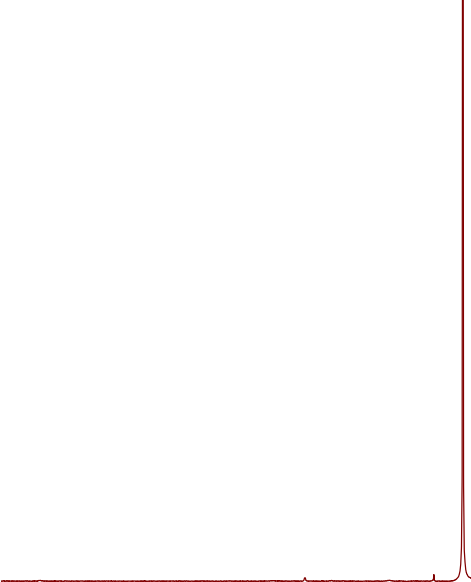

莒

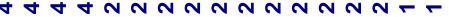

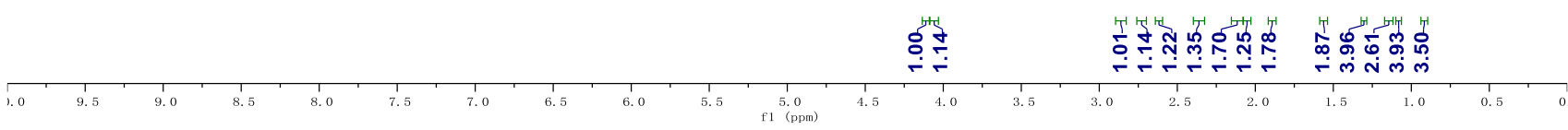

${ }^{13}$ C NMR

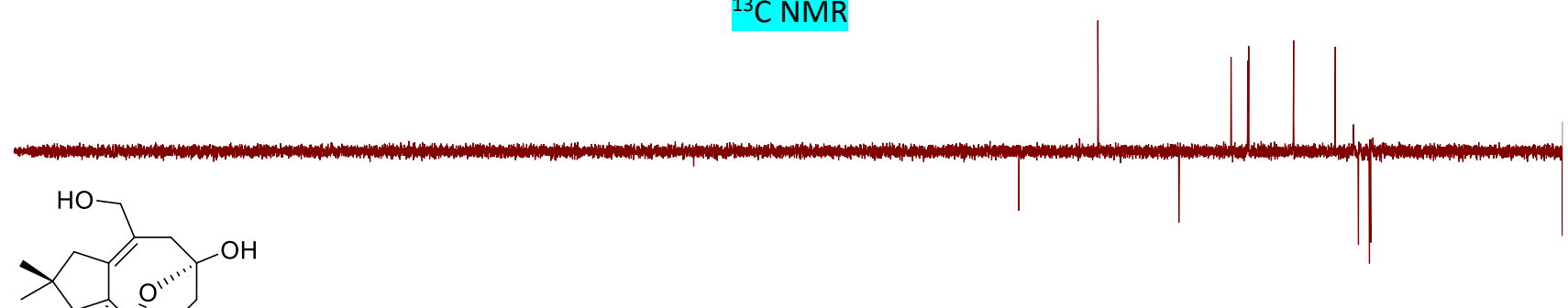

Hi

conosiligin B (2)

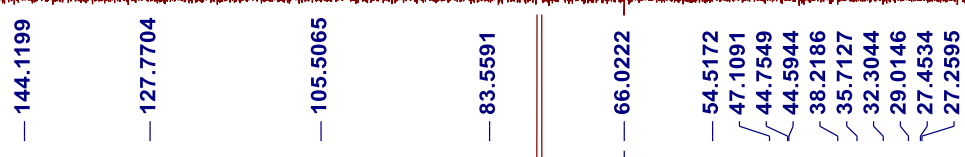

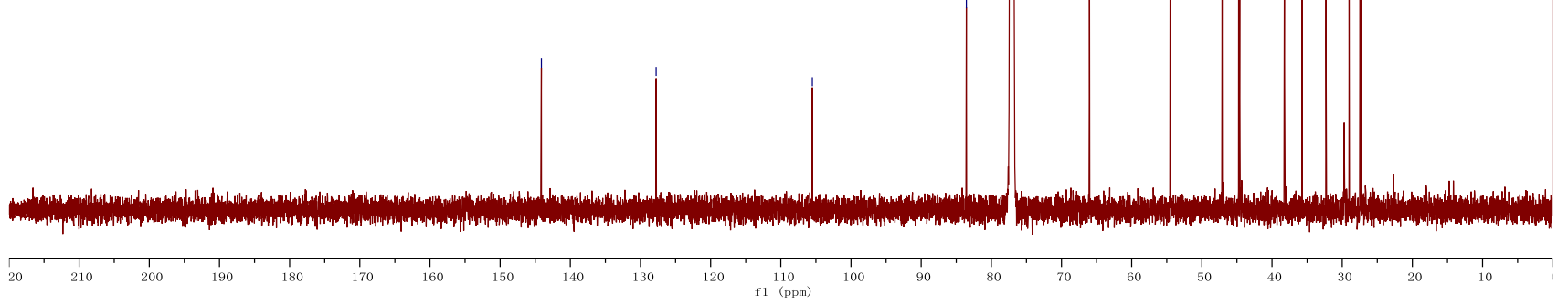


conosiligin B (2)

HSQC

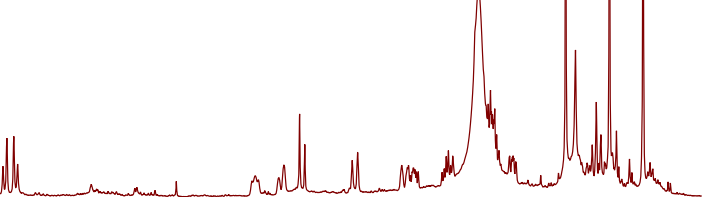

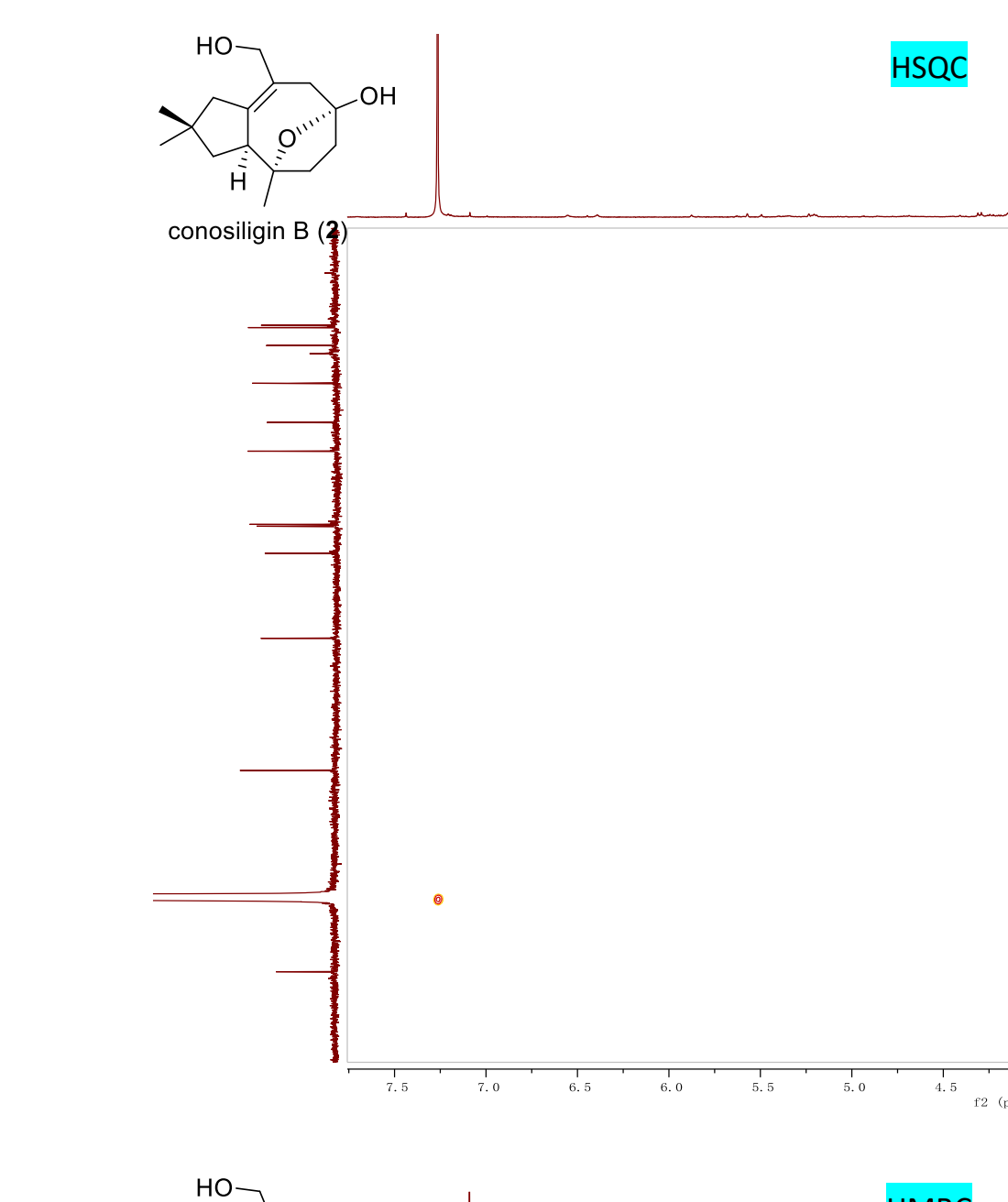
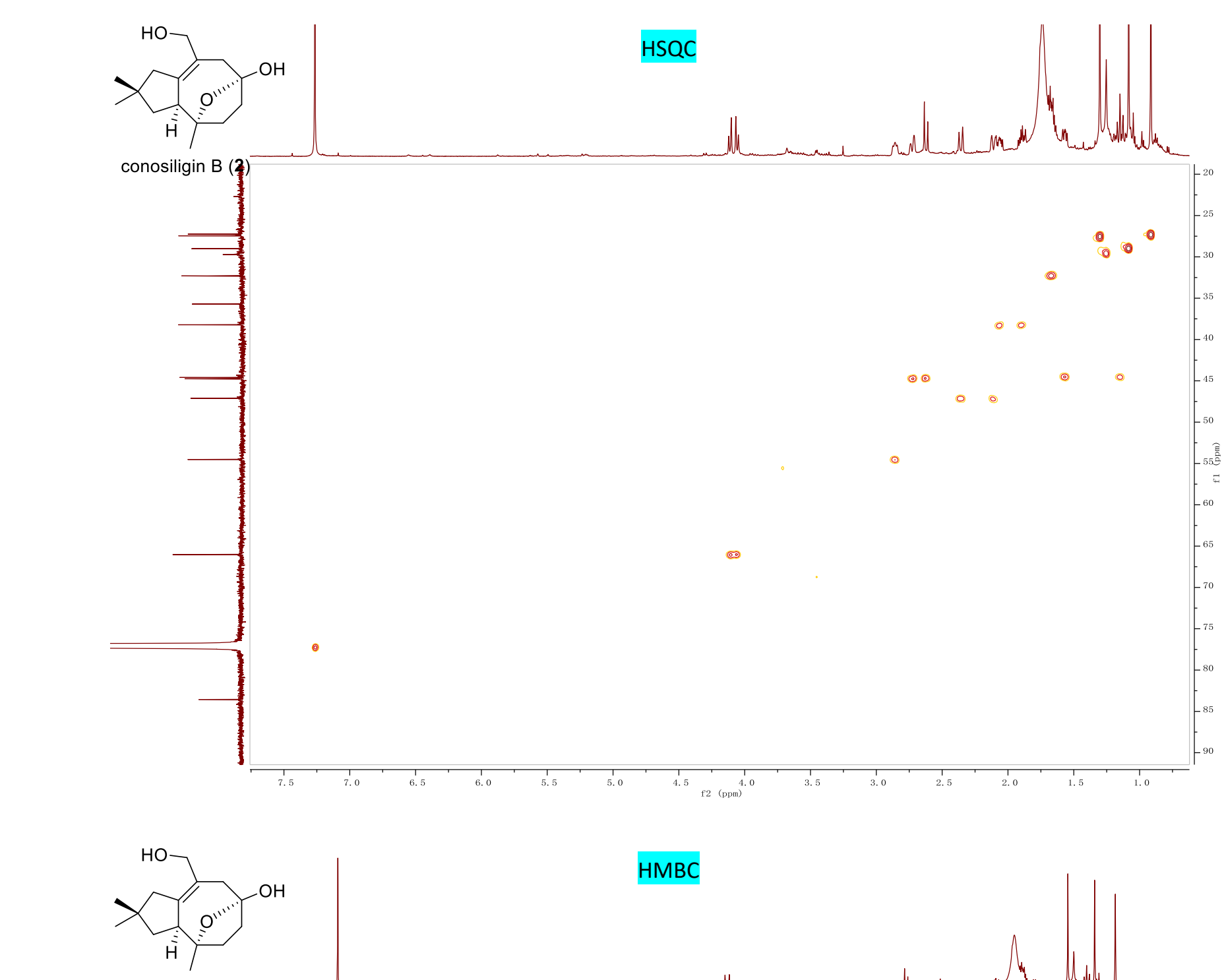

conosiligin B (2)

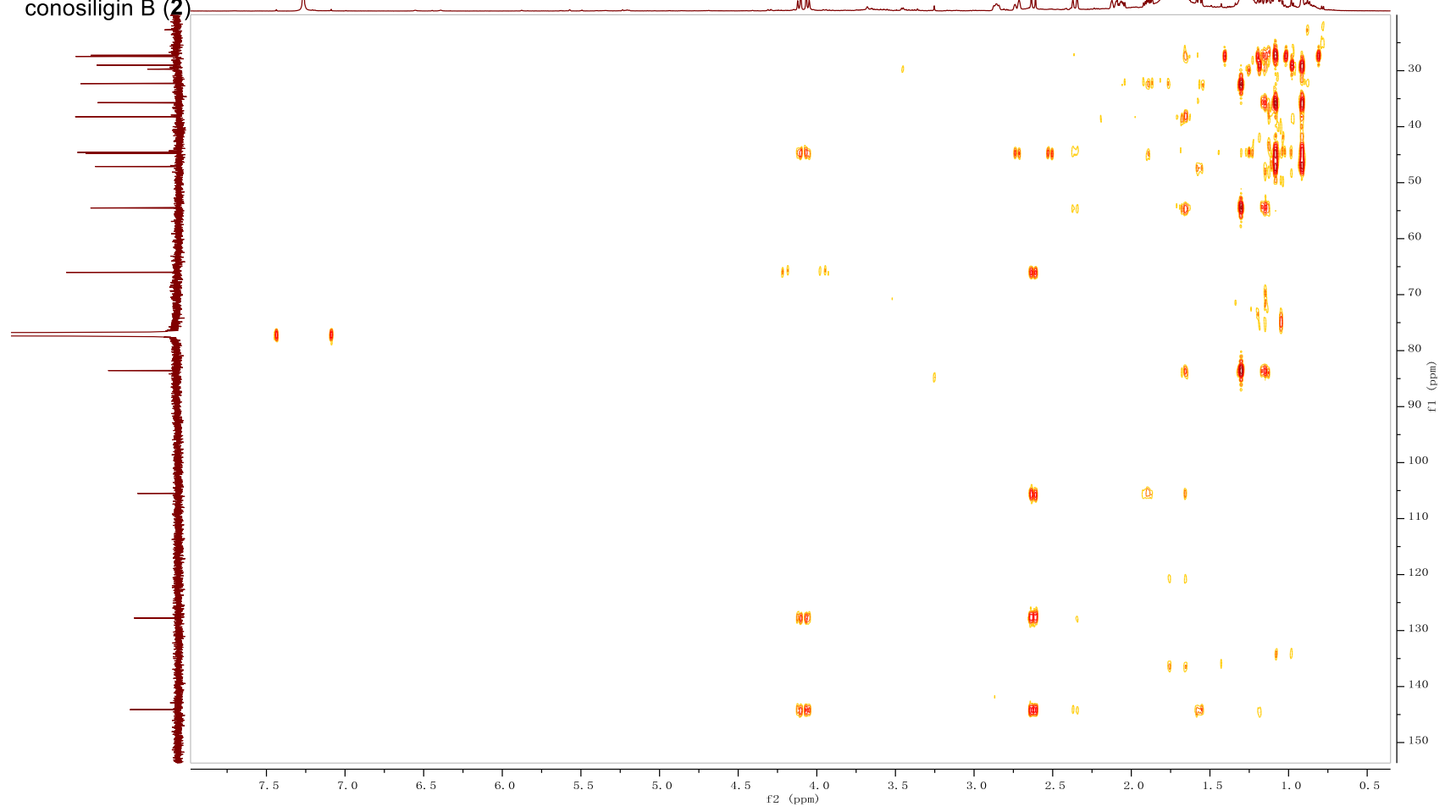

HMBC
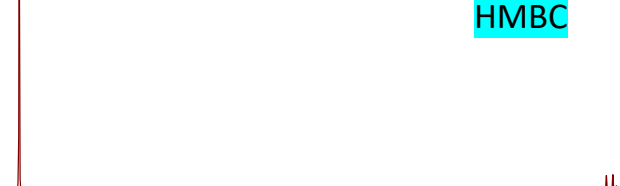
(O)

conosiligin B (2)

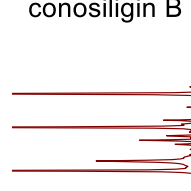

Wh

()

${ }^{1} \mathrm{H}-{ }^{1} \mathrm{H}$ COSY

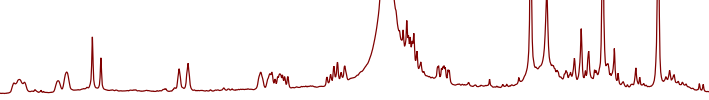

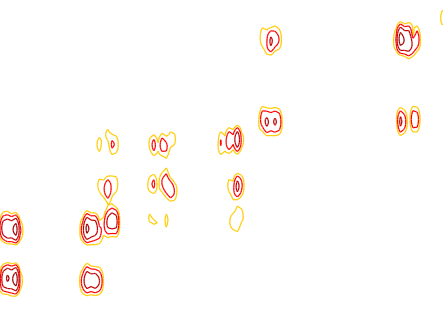

(a)

(0)

00

(90)<smiles>CC1(C)CC2=C(CO)CC(O)(O)C[C@H]3CC21O3</smiles>

\section{ROESY}

$\mathrm{H}$

conosiligin B (2)

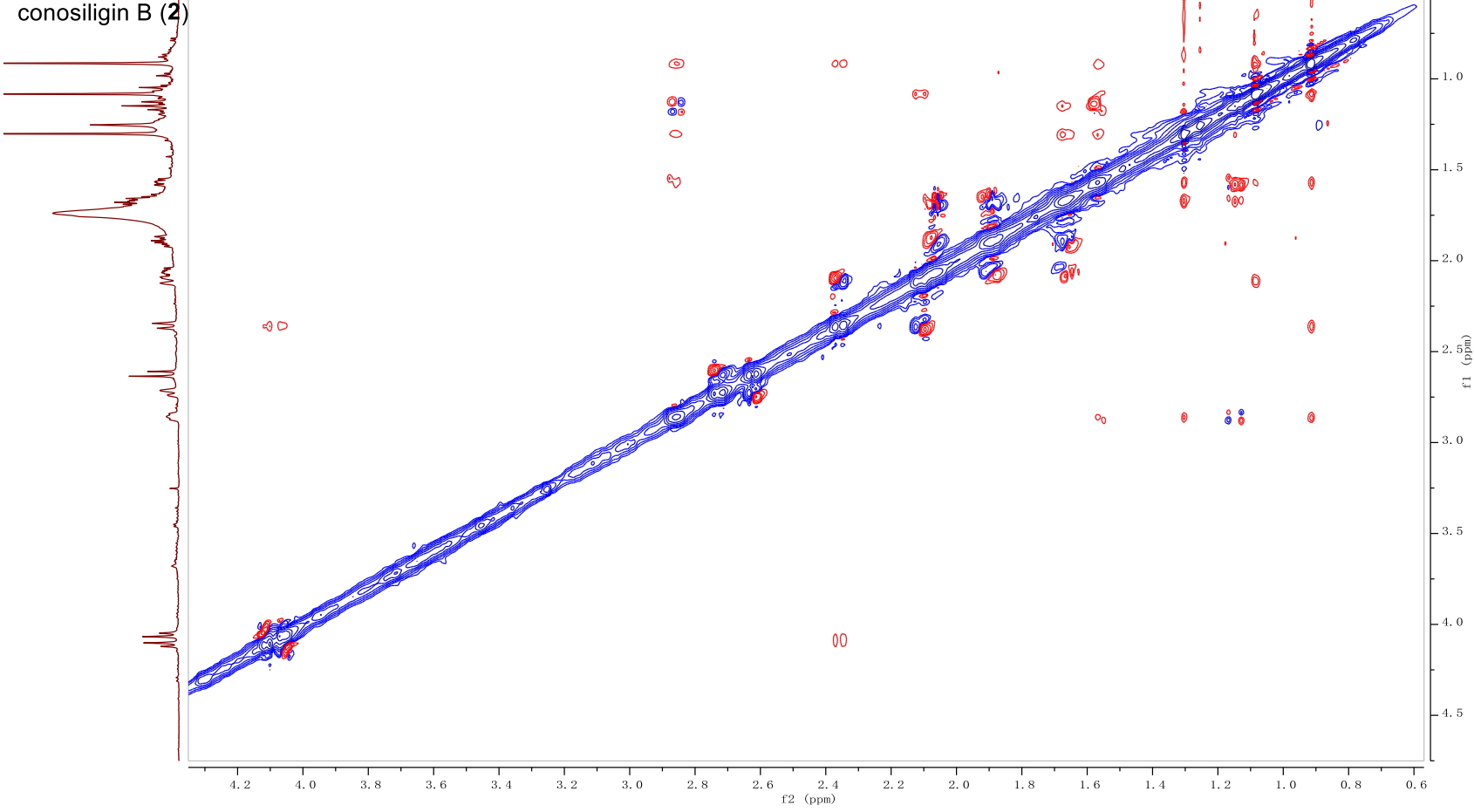




\section{HRESIMS}

T: FTMS + p ESI Full lock ms [150.0000-1100.0000]

253.17975

$\mathrm{C}_{15} \mathrm{H}_{25} \mathrm{O}_{3}$

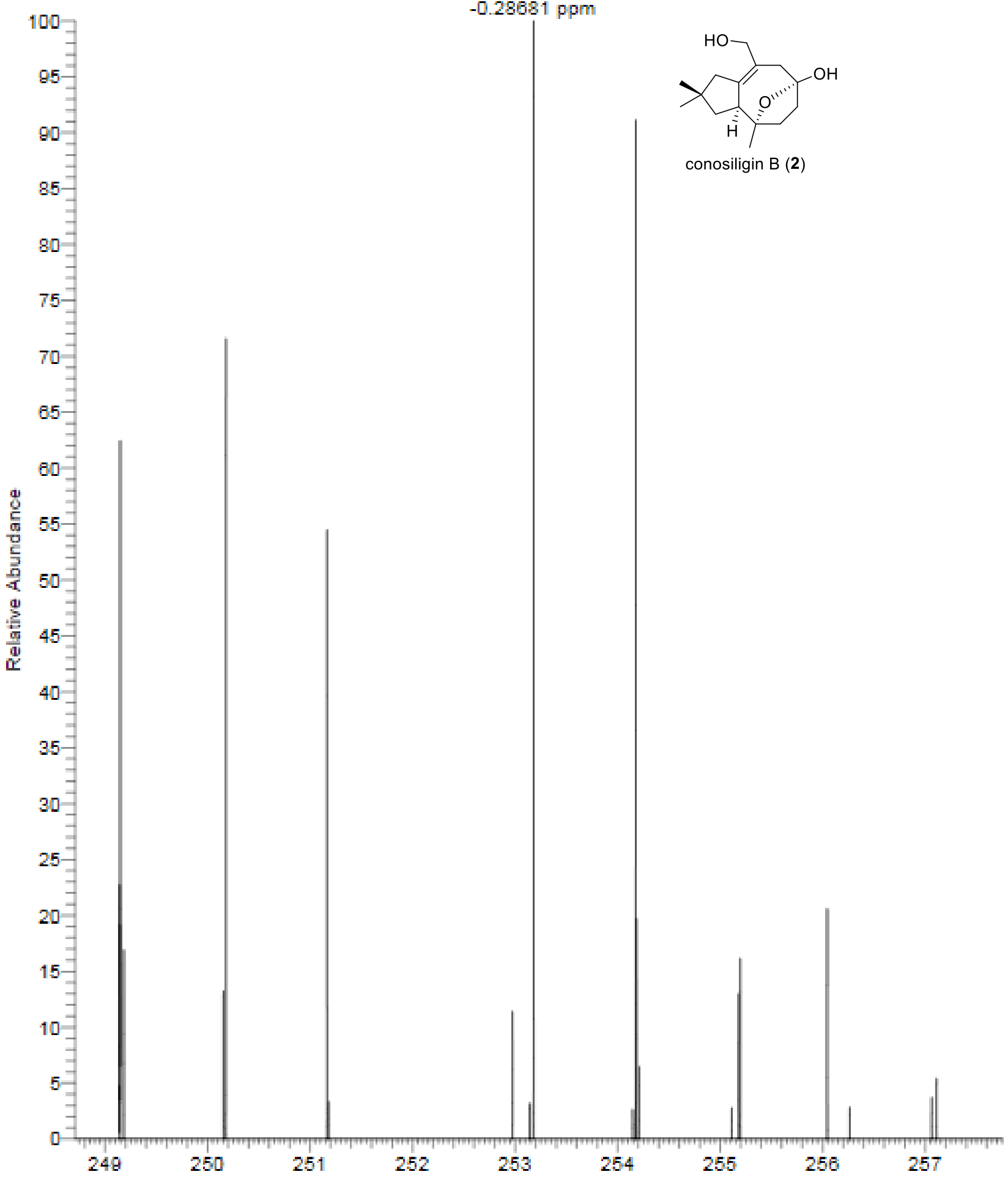


S3.3. NMR spectra (methanol- $d_{4}$ ) and HRESIMS for conosiligin C (3)

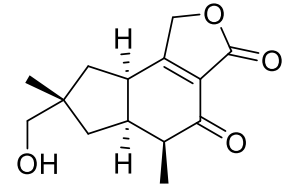

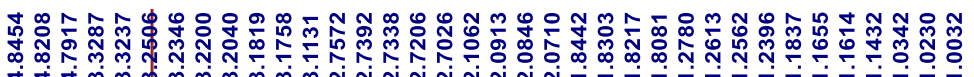

conosiligin $\mathrm{C}(3)$

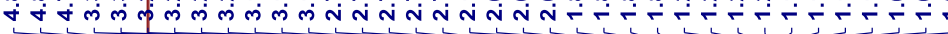

\section{${ }^{1} \mathrm{H} N M R$}
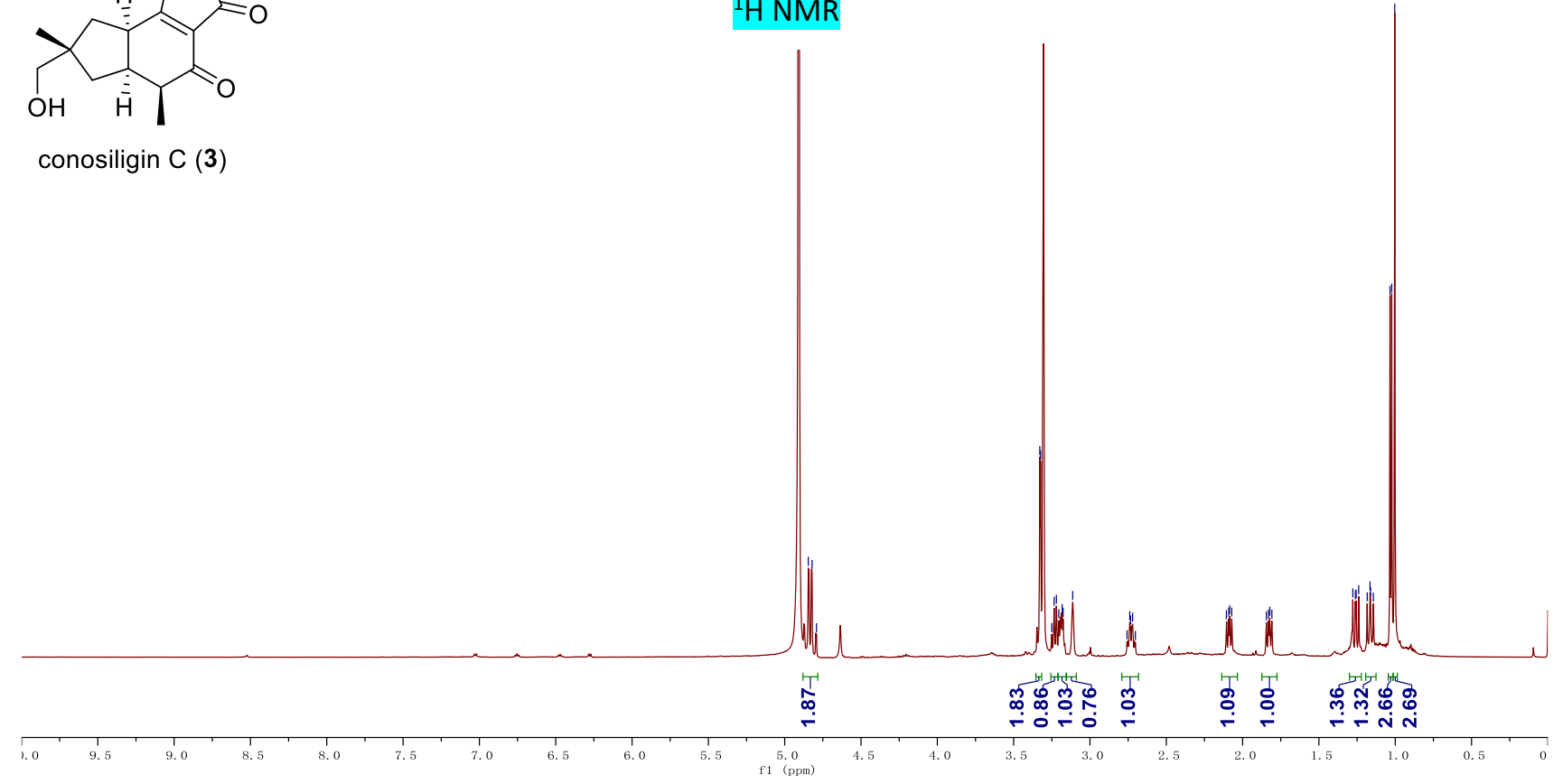

\section{${ }^{13} \mathrm{CNMR}$}
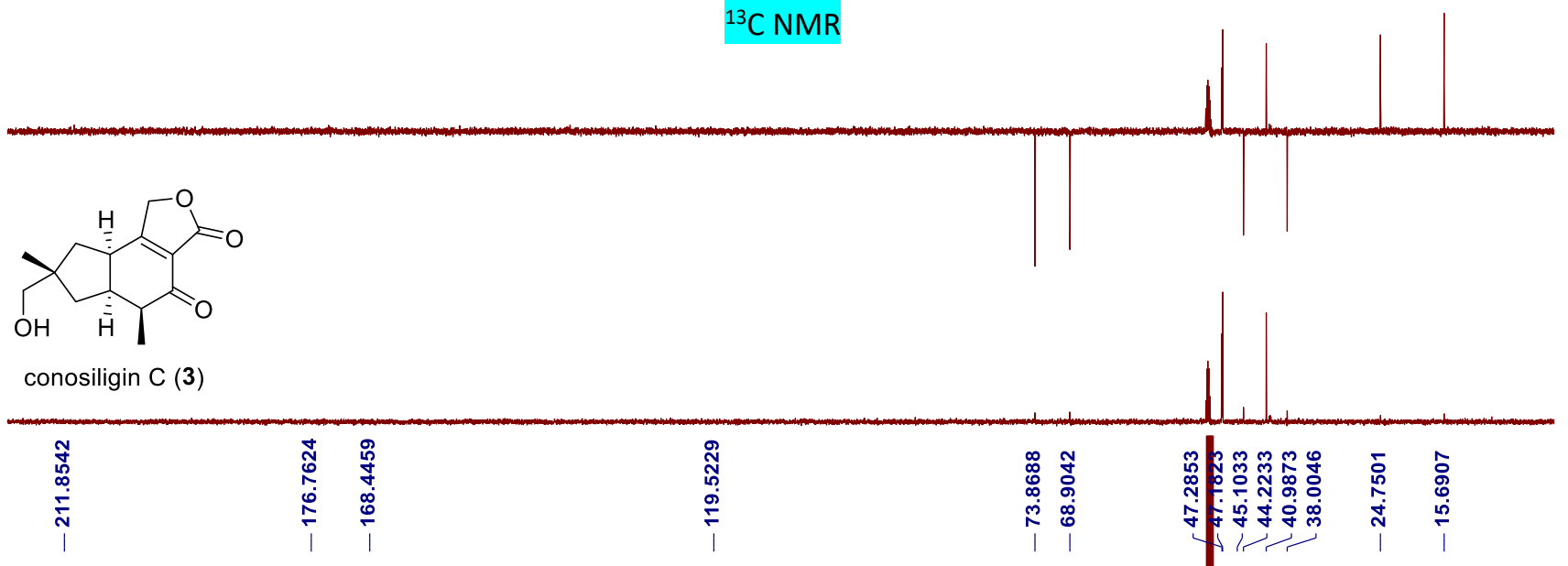

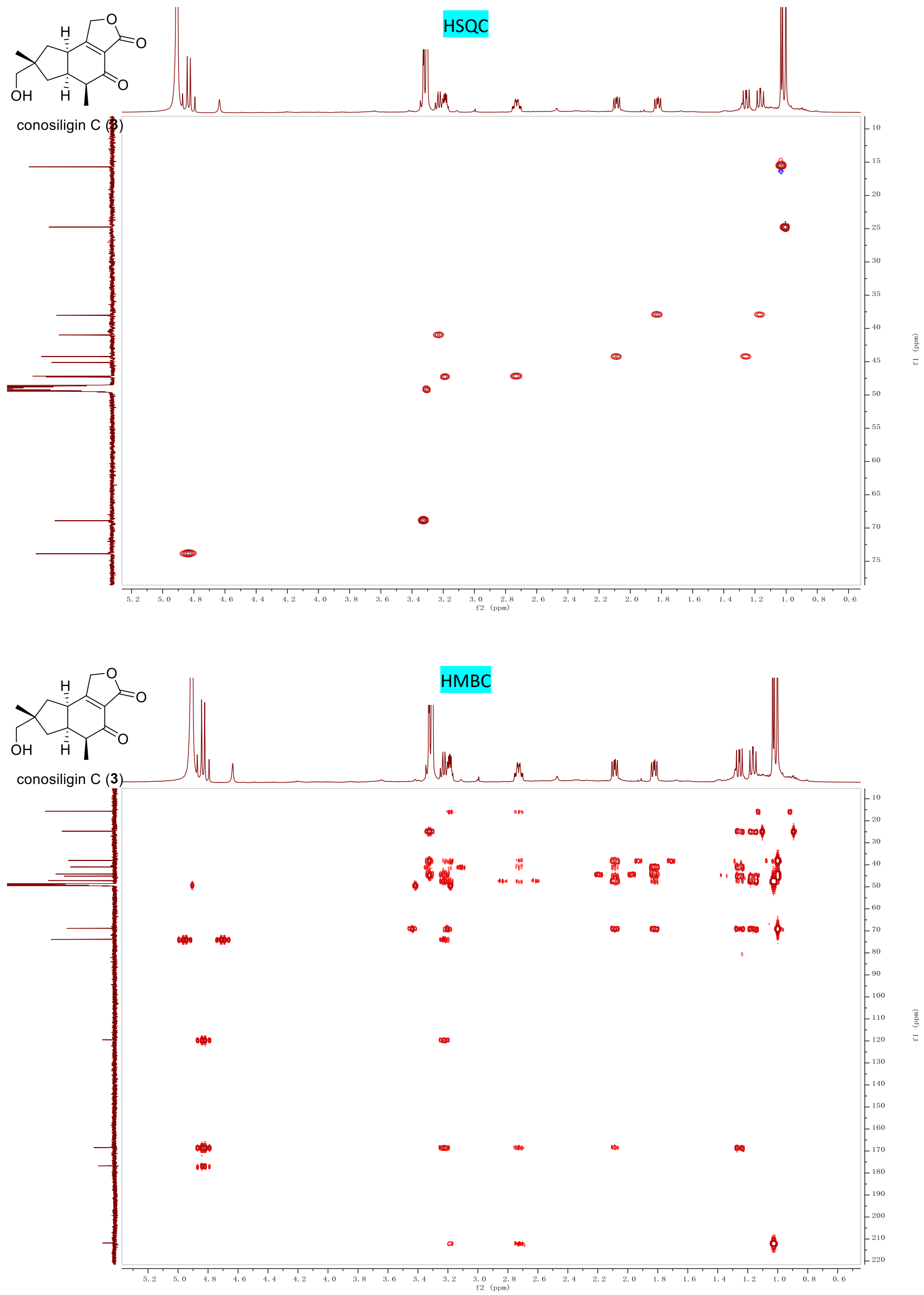

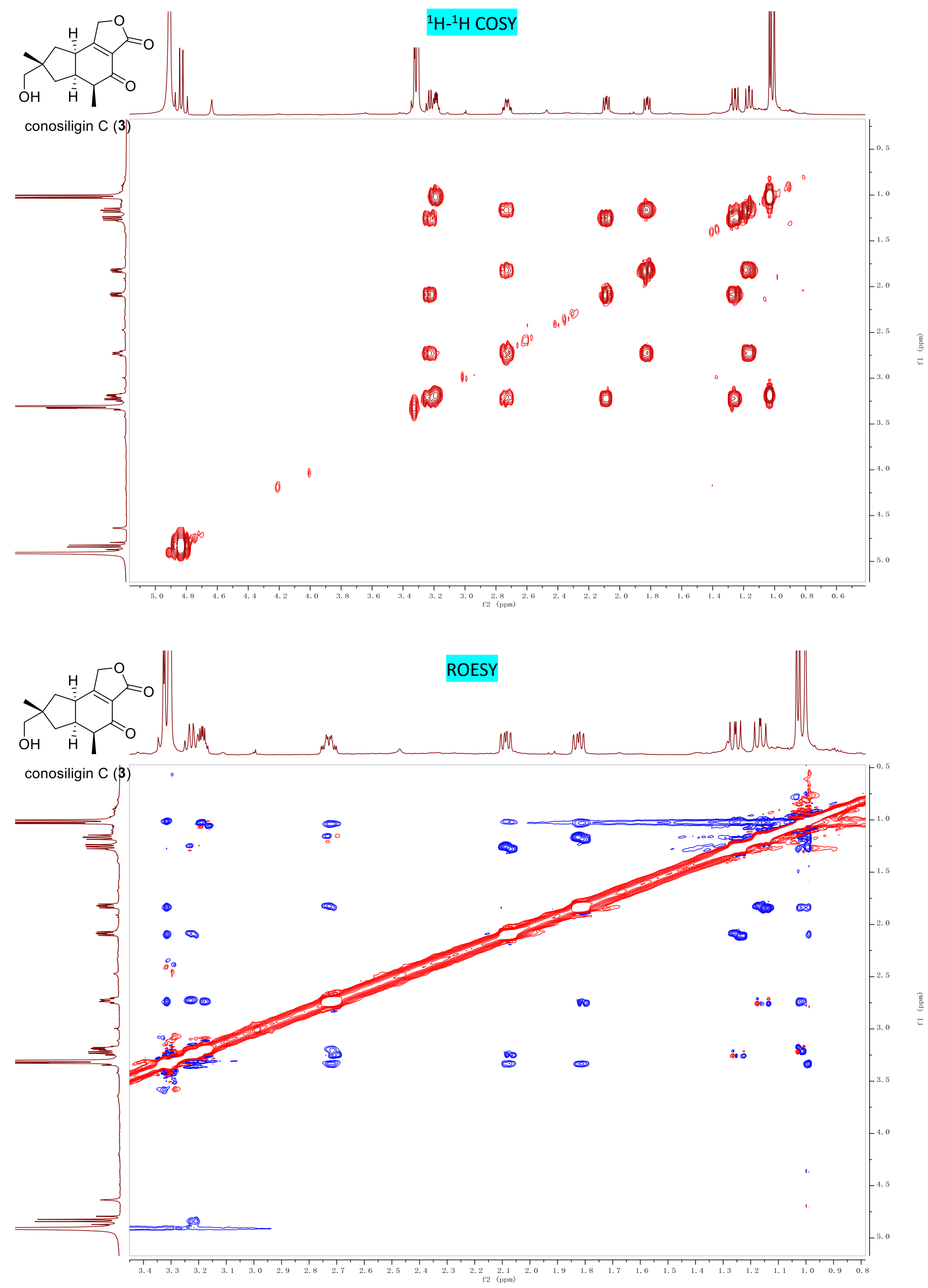
HRESIMS

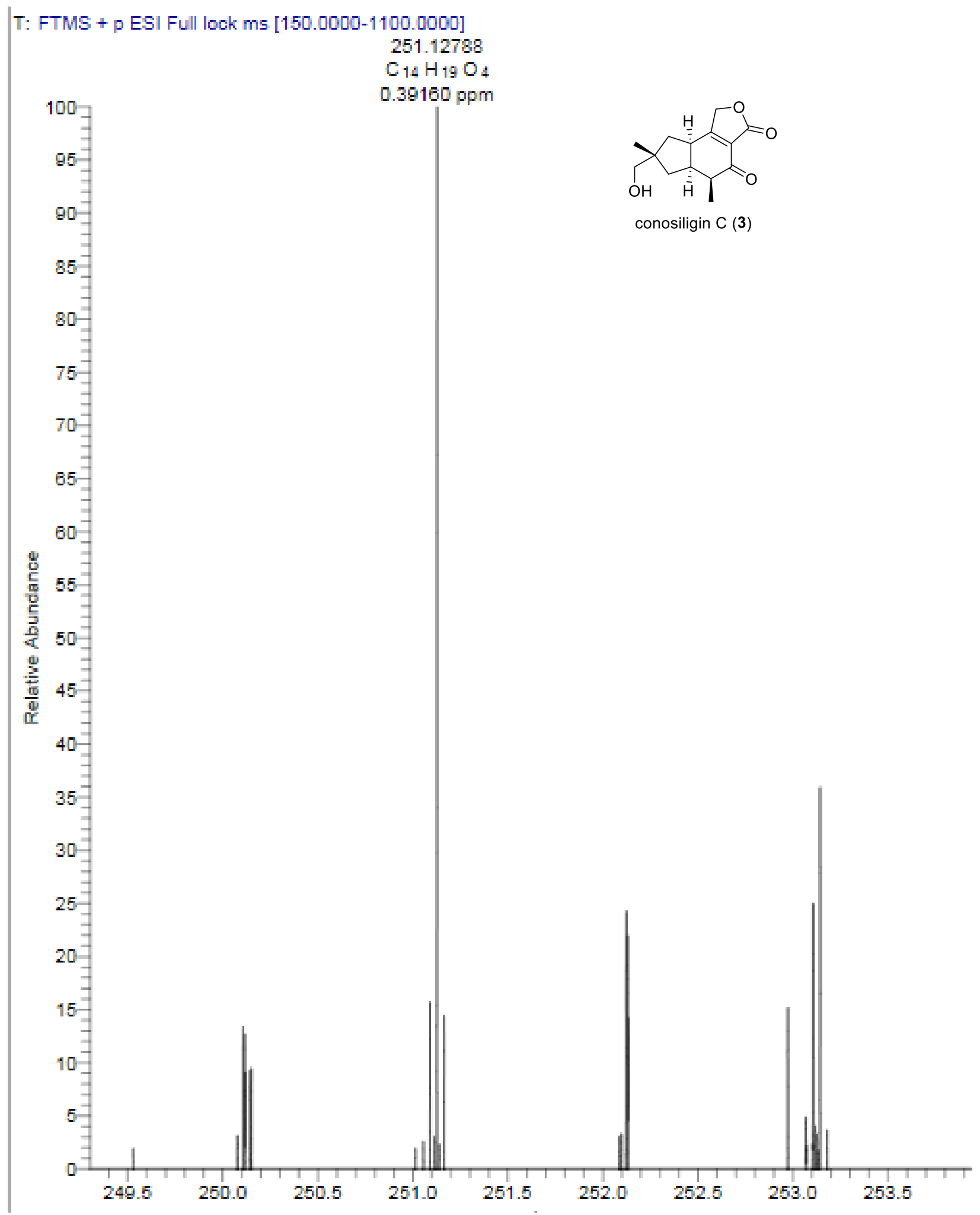


S3.4. NMR spectra (methanol-d $d_{4}$ ) and HRESIMS for conosiligin D (4)
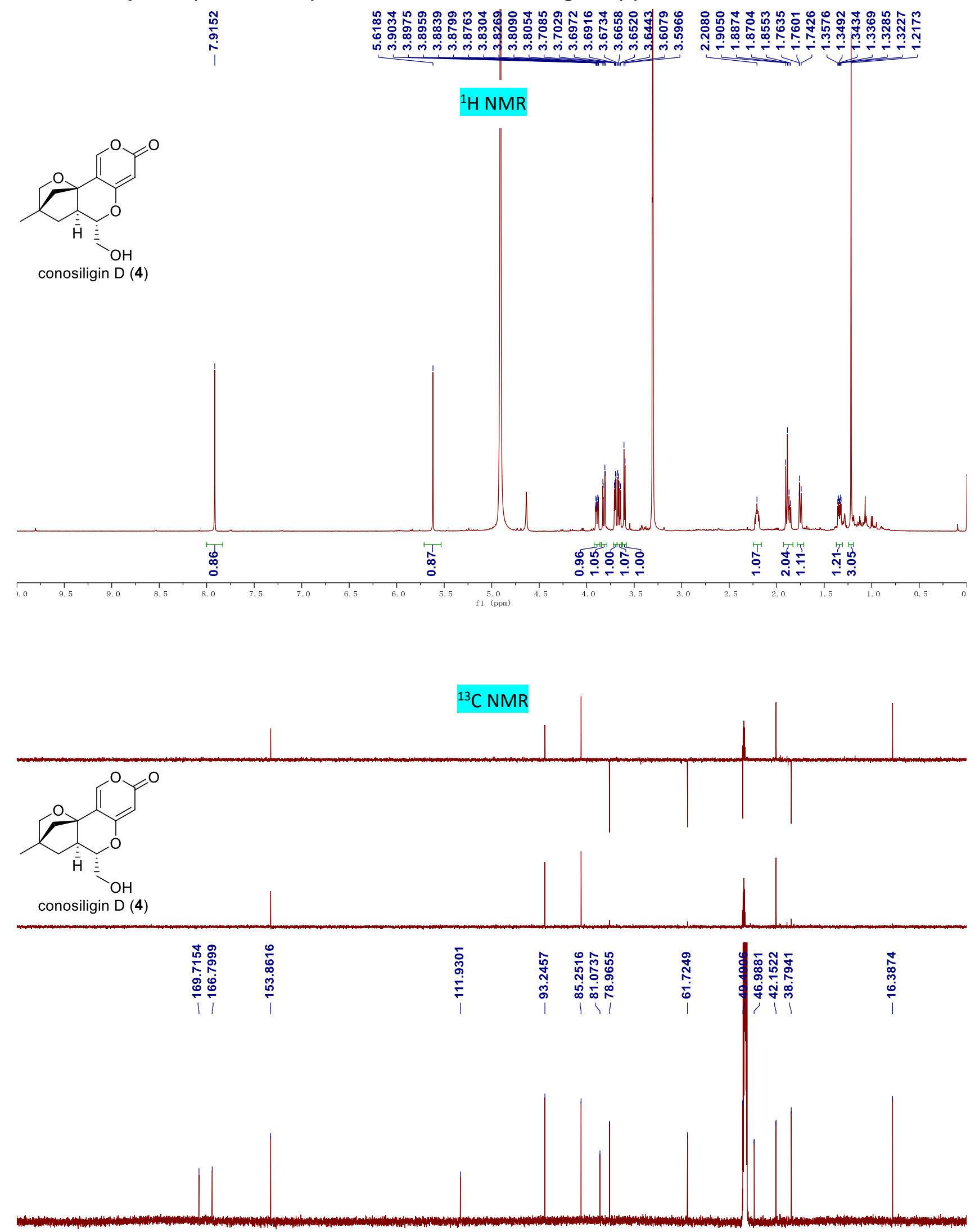

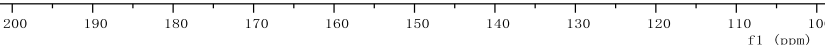



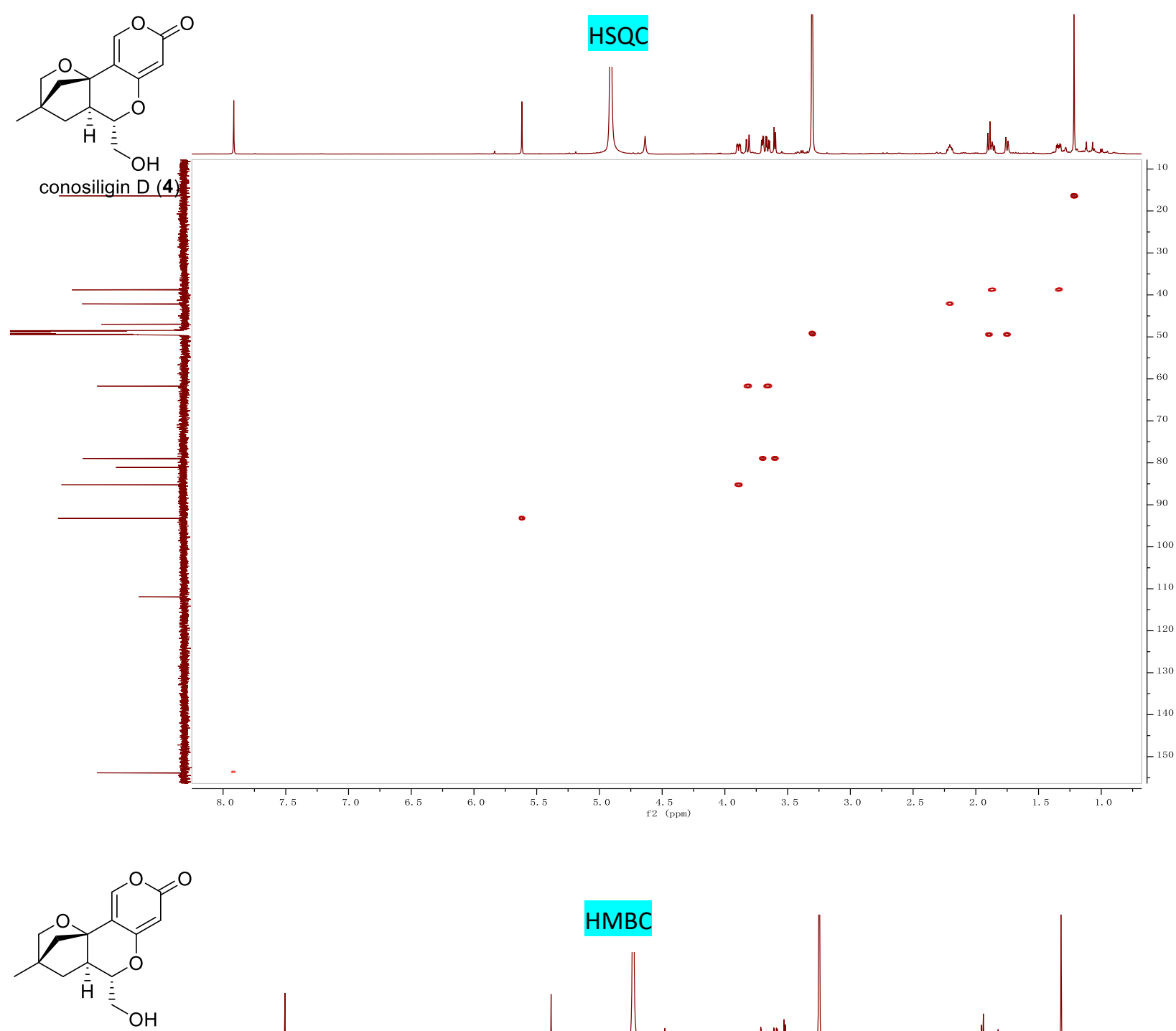

conosiligin $D(4)$

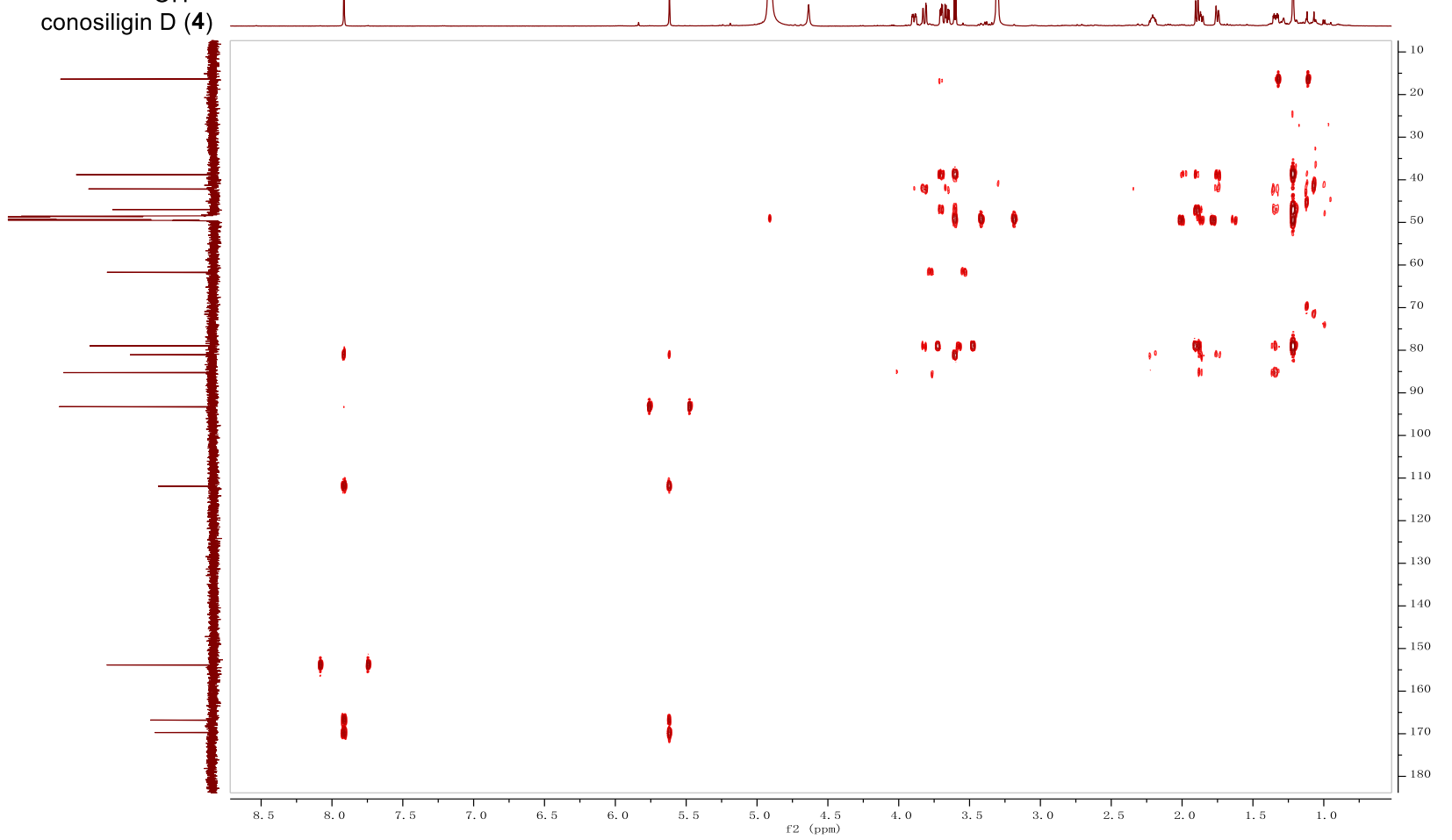



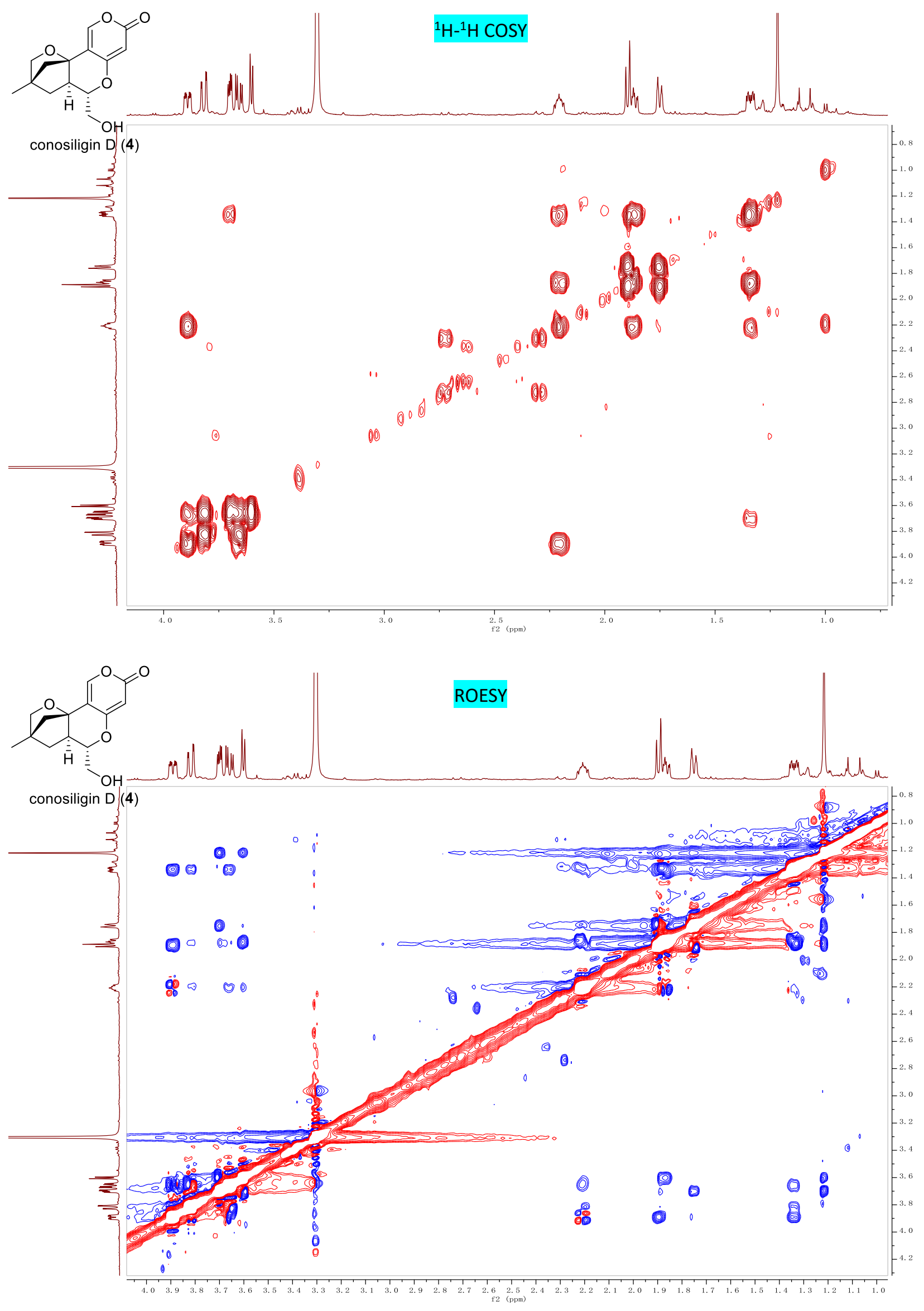


\section{HRESIMS}

T: FTMS + p ESI Full lock ms [200.0000-1100.0000]

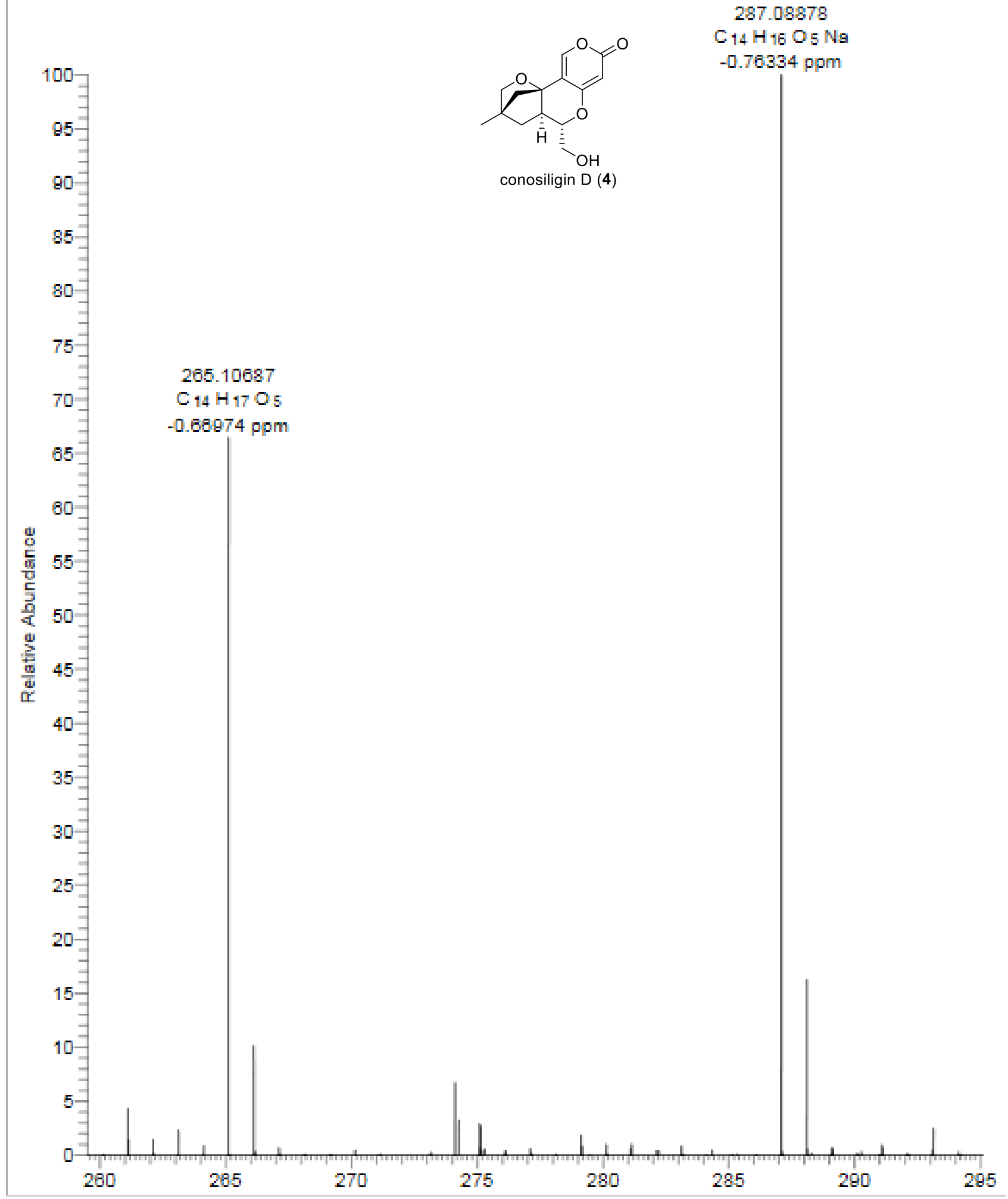

